\title{
Market-Based Estimation of Stochastic Volatility Models*
}

\author{
Yacine Aït-Sahalia ${ }^{\dagger}$ \\ Department of Economics
}

Dante Amengual ${ }^{\ddagger}$

CEMFI

Elena Manresa ${ }^{\S}$
CEMFI

Princeton University and NBER

This Version: May 15, 2014

\begin{abstract}
We propose a method for estimating stochastic volatility models by adapting the HJM approach to the case of volatility derivatives. We characterize restrictions that observed variance swap dynamics have to satisfy to prevent arbitrage opportunities. When the drift of variance swap rates are affine under the pricing measure, we obtain closed form expressions for those restrictions and formulae for forward variance curves. Using data on the S\&P500 index and variance swap rates on different time to maturities, we find that linear mean-reverting one factor models provide inaccurate representation of the dynamics of the variance swap rates while two-factor models significantly outperform the former both in and out of sample.
\end{abstract}

Keywords: HJM approach, Variance swaps, Maximum likelihood estimation.

JEL: G12, C32, C58

\footnotetext{
${ }^{*}$ We would like to thank participants at the Implied Volatility (Huntington Beach) and Measuring Risk Conferences (Princeton) for helpful comments and suggestions.

${ }^{\dagger}$ Research supported by the NSF under grant NES-0850533. Bendheim Center for Finance, 26 Prospect Avenue, Princeton NJ 08540, USA.Email: yacine@princeton.edu

${ }^{\ddagger}$ Centro de Estudios Monetarios y Financieros, Casado del Alisal 5, Madrid, 28014, Spain. Email: amengual@cemfi.es

${ }^{\S}$ Centro de Estudios Monetarios y Financieros, Casado del Alisal 5, Madrid, 28014, Spain. Email: manresa@cemfi.es
} 


\section{Introduction}

We propose in this paper a novel method for estimating stochastic volatility models by adapting the approach of Heath et al. (1992) (HJM) from bond pricing to stochastic volatility models, using volatility derivatives as the market inputs. The HJM approach is motivated by the fact that in liquid markets, such as many markets for options and variance derivatives, one can rely on the observed market prices of standard liquid securities, which are expected to be fair under standard competitive markets assumptions, in order to estimate a model and subsequently price and hedge other more complex or less liquid securities.

In the early option pricing literature, starting with the seminal contributions of Black and Scholes (1973) and Merton (1973), stock prices were assumed to follow univariate processes with constant volatility. The empirical observation that options' implied volatility changes through time, across strike prices and maturities, rapidly gave rise to models relaxing this assumption, such as the stochastic volatility model of Hull and White (1987), or deterministic time-varying models for implied volatilities such as Derman and Kani (1994), Dupire (1993) and Dupire (1994). Stein and Stein (1991) and Heston (1993), among others, developed stochastic volatility models in which innovations to volatility need not be uncorrelated with innovations to the price of the underlying asset, unlike Hull and White (1987). These models proved useful in terms of explaining some of the joint time-series behavior of stock and option prices.

Estimating stochastic volatility models poses substantial challenges, though. Most of the difficulties arise from the fact that, by nature, volatility dynamics are not entirely observable. Further, the presence of one or more additional state variables that drive the volatility of the underlying asset price confers less tractability to the model from an analytic perspective.

In order to overcome the fact that volatility is unobservable, market-based perspectives have been adopted in the stochastic volatility literature. These methods take as primitives a set of financial securities which are liquidly traded. The basic assumption is that the fair price of each security is observable, and any quantity of the security can be sold or bought at its observed price at any point in time. These prices encapsulate what the market is telling the modeler. The market-based modeling approach postulates dynamic equations for the prices of the liquid instruments. The difficulty inherent in the approach consists in checking that the multitude of equations does not introduce inconsistencies and spurious arbitrage opportunities in the model: in other words, if the model is used to price the securities that served as its inputs, does it output back their prices?

Stochastic implied volatility models are an example of market-based models whose primitives are implied volatilities. This type of models focuses on the stochastic dynamics of either a single implied volatility (e.g. Lyons (1997)), the term structure of implied volatility (as in Schönbucher (1999)), or the whole volatility surface (e.g. Albanese et al. (1998) and Ledoit et al. (2002)). While these approaches deal with the problem of stochastic implied volatility from a theoretical perspective, Rosenberg (2000) and Cont et al. (2002), among others, focus on the empirical aspects of the problem. However, estimating 
an arbitrage-free model of stochastic implied volatility is involved since the quantity of interest inherits the nonlinearities of the Black-Scholes formula (see e.g. Durrleman (2004) and Bergomi (2005)).

This paper contributes to the literature on stochastic volatility models by introducing the use of variance swap rates, which are the most actively traded variance related derivative products, instead of implied volatilities, as primitives for the stochastic volatility model, and then by deriving the constraints on the model to make it consistent with its inputs, detailing analytically the class of consistent models, and developing a closed-form likelihood-based estimation procedure for any consistent model.

A variance swap is a forward contract on the future quadratic variation that pays, at expiration, the difference between the quadratic variation over the horizon of the contract and the fixed variance swap rate. The use of variance swap rates offers several advantages for stochastic volatility modeling. In the first place, they are popular volatility derivatives, frequently used by market practitioners as a hedging tool for volatility risk, and have been actively traded in over-the-counter markets since the late 1990s, and in the simpler form of variance futures on exchanges. For that reason, variance swaps constitute a particularly reliable building block for a market based approach. Moreover, they can be easily sythetized from option prices (see e.g. Carr and Wu (2009) and Egloff et al. (2010) among others). ${ }^{1}$ Second, for model tractability, they are especially convenient because of the linearity of their payoffs, together with the fact that variance derivatives can be seen as a family of conditional expectations over the distribution of the underlying's volatility, leading to linear relationships between the unobserved spot variance and the observed rates.

Of course, the model needs to be made consistent in the sense that if the model were used to price its inputs as any other derivative contracts, the model's output price should match the inputed price. We adopt a Markovian setup as in Bühler (2006) as our starting point. For an arbitrary number of sources of uncertainty in the economy, and assuming that we observe as many different time-to-maturity variance swap rates as sources of uncertainty, we characterize in analytical form the restrictions that (observed) derivative prices dynamics must satisfy in order to prevent arbitrage opportunities under the pricing measure. Moreover, we can implicitly define consistent forward variance curves. Consistent dynamic models are crucial for hedging purposes, in particular, where it is more important to fit the time series properties of the variables rather than the time to maturity or strike dimension of implied volatilities. $^{2}$

When, additionally, (i) drift functions of variance swap rates are affine under the pricing measure $Q$, and (ii) forward variance curves are also affine in variance swap rates, we are able to obtain closed-form analytical expressions for the restrictions that affect the dynamics of observed prices and formulae for consistent forward variance curves. In particular, for a model with $d$ sources of volatility risk, we find

\footnotetext{
${ }^{1}$ In practice, they may be a better choice than option prices since deep in- or out-of-the-money options tend to be illiquid. Using variance swap prices avoids this problem because prices of individual options are aggregated, so the impact of liquidity-induced idiosyncratic errors in each option price is reduced.

${ }^{2}$ The usual practice relies on calibrating alternative models using information of stock and option prices (equivalently implied volatilities) at a given point in time, which ultimately is not accounting for the dynamics of the processes.
} 
that a consistent affine-drift model is determined by $d+1$ free parameters. These parameters can be naturally interpreted as speeds of mean reversion of the stochastic system and its steady state level.

Apart from its tractability, the affine- $Q$ drift models have two additional attractive features worth mentioning. First, the diffusion functions of variance swap rates remain unrestricted except for the instantaneous variance of the underlying asset; hence, leaving room for non-affine specifications of the diffusion such as the constant elasticity of variance (CEV) model of Jones (2003), which is a relevant feature when fitting the models to the data; see, e.g., Li and Zhang (2013). Second, drift specifications richer than the ones belonging to the affine class can be obtained under the objective measure $P$ through different specifications of market prices of risk.

For estimation, we rely on observations on the joint time-series of the underlying asset price and several variance swap rates with different maturities. Our joint modelling strategy allows us to separately pin down risk premia related to each of the different sources of uncertainty. However, as is usual in many financial applications dealing with multivariate diffusions, likelihood functions are not known explicitly for the models of interest. The solution to this problem relies on the approach of Aït-Sahalia (2008), who developed closed-form series expansions for the likelihood function for arbitrary multivariate continuoustime diffusions at discrete intervals of observations. Moreover, maximum likelihood estimation allows us to use likelihood ratio tests to evaluate the fit of non-nested models (see Vuong (1989)), which is difficult or impossible for other methods, such as the method of moments.

We derive explicit pricing formulae for two important classes of affine- $Q$ drift models. The first one includes two sources of uncertainty in the economy, one specific to variance swap rates and one common to both variance swap rates and the stock; the second one assumes there are three sources of uncertainty, two of which driving the dynamics of variance swap rates. The first class of models we derive nests several stochastic volatility models already considered in the literature such as Heston (1993)'s, the GARCH stochastic volatility model considered in Nelson (1990), or the CEV model of Jones (2003). The second one generalizes stochastic volatility specifications with two volatility factors considered in Gatheral (2008), Amengual (2008), Christoffersen et al. (2009) and Egloff et al. (2010) among others, and is compatible with the evidence in favor of two volatility factors provided by Li and Zhang (2010).

We assess the small sample properties of the parameter estimates resulting from our proposed methodology by means of Monte Carlo experiments. Given the numerical tractability of the model and estimation method, we can easily perform large numbers of Monte Carlo simulations. In particular, we examine different scenarios including the effect of using different information sets, sampling frequency, near unit-root behavior for volatility and compare the small sample behavior of the estimators to their predicted asymptotic one. As expected, the sampling variance of diffusion parameters estimates is smaller than the corresponding to drift parameters, specially for those entering only through the $P$ measure. Finally, with additional variance swap rates, the precision of the $Q$-drift parameters estimates significantly increases. 
When taking the models to the data, we find that observation of additional variance swap rates with different time-to-maturity rates lead to significant efficiency gains in the estimation of the drift parameters under the pricing measure. Moreover, in agreement with the recent option pricing literature, our empirical results suggest that linear mean-reverting one factor models provide inadequate representation of the dynamics of the stochastic volatility. In contrast, models where two factors drive the volatility dynamics provide a better fit to the data, both in and out of sample, while allowing for richer term structures of forward volatilities (a feature also noted in Bates (2000), Amengual (2008) and Egloff et al. (2010)).

The rest of this paper is organized as follows. Section 2 derives consistent variance swap models in which as many variance swap rates as sources of uncertainty driving the volatility dynamics are being directly modeled. We also characterize the families of risk neutral-drift affine models that prevent arbitrage opportunities. The estimation methodology is presented in Section 3, while Section 4 contains an empirical estimation of the one- and two-factors variance swap models to S\&P500 data. Section 5 concludes. Proofs are gathered in Appendix 1. The Supplemental Appendix 2 reports results on the finite sample performance of the estimation method for the consistent models we derived.

\section{Market-based variance curve models}

The forward variance curve is the mapping from time-to-maturity to forward variance for each possible state in the economy. In this section, we characterize the dynamics of families of arbitrage-free variance curves in terms of variance swap rates. We derive analytical closed-form solutions for particular families in which the drift functions of variance swap rates are affine- $Q$. Finally, we provide examples when the dynamics of one and two variance swap rates are modeled jointly with the dynamics of the underlying.

\subsection{Primitives and model specification}

We consider a continuous trading economy with a trading interval $[0, \mathcal{T}]$ for a fixed $\mathcal{T}>0$. We characterize the uncertainty in the economy by the probability space $(\Omega, \mathcal{F}, P)$ where $\Omega$ is the state space, $\mathcal{F}$ is the $\sigma$-algebra representing measurable events, and $P$ is the objective probability measure. Information evolves over the trading interval according to the augmented, right continuous, complete filtration $\left(\mathcal{F}_{t}^{P}\right)_{t \geq 0}$ generated by $d+1$ independent Brownian motions $\left\{W_{0 t}^{P}, \ldots, W_{d t}^{P}: t \in[0, \mathcal{T}]\right\}$ initialized at zero. We define $\mathbf{W}^{P}=\left(W_{0}^{P}, W^{P \prime}\right)^{\prime}$ where $W^{P}=\left(W_{1}^{P}, \ldots, W_{d}^{P}\right)$. All processes are adapted with respect to $\left(\mathcal{F}_{t}^{P}\right)_{t \geq 0}$. We denote by $\mathcal{L}^{2}$ the space of all adapted square-integrable processes.

Let $S$ be the stock price process and $\sigma$ the spot volatility process and assume $\int_{0}^{t} \sigma_{s}^{2} d s<\infty$ a.s. Assume $W_{0}^{P}$ is specific to $S$; that is, $\sigma$ is measurable with respect to the filtration generated by $W^{P}$ only. ${ }^{3}$ We complete the model by assuming that at least $d$ variance swap rates $Y_{t}^{d}=\left(Y_{t}^{\tau_{1}}, \ldots, Y_{t}^{\tau_{d}}\right)^{\prime} \in \mathcal{Y}$ with different time-to-maturities $\left(\tau_{1}, \ldots, \tau_{d}\right) \in \mathbb{R}_{+}^{d}$ are traded.

\footnotetext{
${ }^{3}$ Note that this assumption is just a matter of parameterization and does not involve relevant restrictions for the models we consider: it can be obtained by rotation of the primitive stochastic factors.
} 
From the well-known results of Harrison and Kreps (1979) and Harrison and Pliska (1981), and many extensions since then, the existence of an equivalent martingale measure $Q$ guarantees the absence of arbitrage among a broad class of admissible trading strategies. For simplicity, we fix to zero the instantaneous interest rate, $r$, as our analysis applies in the context of deterministic interest rates in order to limit the number of state variables. Therefore, under the $Q$ measure the joint dynamics of $S, Y_{t}^{\tau_{1}}, \ldots, Y_{t}^{\tau_{d}}$ can be written as

$$
\begin{aligned}
d S_{t} & =\sigma_{t} S_{t}\left[\sqrt{1-\|\rho\|^{2}} d W_{0 t}^{Q}+\rho d W_{t}^{Q}\right] \\
d Y_{t}^{\tau_{1}} & =\mu_{Y_{1}}^{Q}\left(Y_{t}^{\tau_{1}}, \ldots, Y_{t}^{\tau_{d}}\right) d t+\Sigma_{Y_{1}}\left(Y_{t}^{\tau_{1}}, \ldots, Y_{t}^{\tau_{d}}\right) d W_{t}^{Q} \\
& \vdots \\
d Y_{t}^{\tau_{d}} & =\mu_{Y_{d}}^{Q}\left(Y_{t}^{\tau_{1}}, \ldots, Y_{t}^{\tau_{d}}\right) d t+\Sigma_{Y_{d}}\left(Y_{t}^{\tau_{1}}, \ldots, Y_{t}^{\tau_{d}}\right) d W_{t}^{Q}
\end{aligned}
$$

where $\rho$ defines the correlation structure of $S$ with its volatility process where $\rho \in[-1,1]^{d}$ with $\|\rho\|<1$

and $\|$.$\| denotes the Euclidean norm. Finally, \mu_{Y_{i}}^{Q}$ is the $i$ 'th element of the drift function $\mu_{Y}^{Q}$ corresponding to $Y_{t}^{d}=\left(Y_{t}^{\tau_{1}}, \ldots, Y_{t}^{\tau_{d}}\right)^{\prime}$, and similarly, $\Sigma_{Y_{i}}$ is a $d \times 1$ vector of the $i$ 'th row of the diffusion matrix $\Sigma_{Y Y}$. For simplicity, we assume that the elements of $\rho$ are constants.

The primitives considered so far aim at building a framework where the choice of dynamic specifications is only required for the observed process, and where the dynamics of the latent factors are recovered through arbitrage restrictions. This imposes, naturally, modelling discipline on the researcher, who is forced to choose among realistic and plausible specifications for the observed process. Our subsequent analysis will now derive the link between observed quantities, the variance swap rates, and the latent factor, the stochastic volatility process.

\subsection{Imposing no-arbitrage restrictions on variance swap rates dynamics}

So far we have stated the model ingredients, namely by specifying the joint dynamics of variance swaps rates as well as the underlying asset, which we call a stock for concreteness. Our purpose now is to derive the constraints that are required in the $Q$ dynamics by imposing no-arbitrage restrictions on the system.

Variance swaps are forward contracts on the future quadratic variation of a stock that pay, at expiration, the difference between its quadratic variation over the horizon of the contract and the fixed variance swap rate. Since we take them as our market primitives, we assume that variance swap contracts are tradable at any point in time and that their prices are fair. Alternatively, one could rely on standard option markets from which variance swaps can be synthetized. Since there is no initial payment involved and given that variance swap rates are liquidly traded, its price at $t$ for a fixed maturity $T, \mathrm{VS}_{t}^{T}$, is given by

$$
\mathrm{VS}_{t}^{T}=\int_{0}^{t} \sigma_{s}^{2} d s+E_{t}^{Q}\left[\int_{t}^{T} \sigma_{s}^{2} d s\right]
$$


By the Martingale Representation Theorem, the dynamics under $Q$ of $\operatorname{VS}_{t}^{T}$ are given by

$$
d \mathrm{VS}_{t}^{T}=B_{t}(T) \cdot d W_{t}^{Q}
$$

for some $B(T) \in \mathcal{L}^{2}$. Similarly to bond pricing, we define the fixed maturity $T$-forward variance at $t$ by

$$
v_{t}(T):=\frac{\partial \mathrm{VS}_{t}^{T}}{\partial T}=E_{t}^{Q}\left[\sigma_{T}^{2}\right], \text { for } \mathcal{T} \geq T, t \geq 0
$$

The interpretation for this quantity is analogous to the forward interest rate in fixed-income analysis. From the uniqueness of the representation of a local martingale on $\left(\mathcal{F}_{t}\right)_{t \geq 0}$ with respect to $W^{Q}, v(T)$ is a non-negative martingale with representation

$$
d v_{t}(T)=b_{t}(T) \cdot d W_{t}^{Q}
$$

with $b_{t}(T):=\partial B_{t}(T) / \partial T \in \mathcal{L}^{2}$. This condition can be interpreted as the "HJM-condition" for variance curves as in Bühler (2006). And analogously to the HJM approach in fixed-income analysis, we assume that for each fixed $t, \operatorname{VS}_{t}^{T}$ is a smooth function of the maturity $T$, and define the forward variance $v_{t}(T)$ as its derivative with respect to maturity.

Our purpose is to impose the no-arbitrage restrictions implied by (2.2) on the dynamics of the fixed time-to-maturity variance swap rates given in (2.1), since this is what matches the available market data on variance swaps. Hence, we need to restate (2.2), which was obtained for fixed maturity $T$, in terms of fixed time-to-maturity. To do so, it is convenient to change the parameterization to $\tau=T-t$. Specifically, we define $Y_{t}^{\tau}$ as the fixed time-to-maturity $\tau$ (annualized) variance swap rate as

$$
Y_{t}^{\tau}=\frac{1}{\tau} E_{t}^{Q}\left[\int_{t}^{t+\tau} \sigma_{s}^{2} d s\right] .
$$

Similarly, we can define the fixed time-to-maturity forward variance by $u_{t}(\tau):=v_{t}(t+\tau)$, in the same spirit of the so-called "Musiela-parametrization" of forward rates. It is useful to think of $u_{t}(\tau)$ as the variance swap rate one can contract at time $t$ on the quadratic variation of $S$ that begins at date $t+\tau$ and is returned an instant later.

Under mild regularity conditions on the process $b,(2.2)$ and the Itô-Wentzell formula allow to describe the dynamics of $u$ as

$$
d u_{t}(\tau)=\frac{\partial u_{t}(\tau)}{\partial \tau} d t+\beta_{t}(\tau) \cdot d W_{t}^{Q}
$$

where $\beta_{s}(\tau):=b_{s}(t+\tau)$. Note also that the spot variance at time $t$ is the instantaneous forward variance rate at time $t$ for zero time-to-maturity, that is, $\sigma_{t}^{2}=u_{t}(0)$.

We define the forward variance curve $G$ as a non-negative $\mathcal{C}^{2,1}$-function taking values on $\mathcal{Y} \times[0, \mathcal{T}]$, with $\mathcal{Y} \subset \mathbb{R}_{+}^{d}$, and with bounded $\int_{0}^{\tau} G(y, s) d s$ for all $(y, \tau)$ in the parameter space.

Given $G$ and $Y_{0}^{d} \in \mathcal{Y}$ and a process $Y^{d}=\left(Y_{t}^{d}\right)_{t \geq 0}$ characterized by (2.1), we define the functional $G$ as $G\left(Y_{t}^{d}, \tau\right):=u_{t}(\tau), \tau \geq 0 .{ }^{4}$ As in Bühler (2006), hence, we characterize the forward variance curve

\footnotetext{
${ }^{4}$ In general, it is very complicated to ensure non-negativity of the function $G$; see Bühler (2006) for a detailed discussion on that issue.
} 
through the instantaneous variance swap rate. ${ }^{5}$ But we need to go further, since our object of interest is not the variance curve - which cannot be related directly to the observed processes-, but the integrals over it, that directly relate to the variance swap rates, i.e. the observed processes.

The following result gives general conditions that provide: (i) restrictions in the dynamics of $Y^{d}$, and (ii) a characterization of the functional form of $G\left(Y^{d}, \tau\right)$, consistent with the model being arbitrage free.

Proposition 1. Let us assume that the joint dynamics of the spot $d \log S_{t}$ and a vector of variance swap rates $Y_{t}^{d}$ with time-to-maturity $\tau_{1}, \ldots, \tau_{d}$ be

$$
\left[\begin{array}{c}
d \log S_{t} \\
d Y_{t}^{d}
\end{array}\right]=\left[\begin{array}{c}
\mu_{S}^{Q}(\cdot) \\
\mu_{Y}^{Q}(\cdot)
\end{array}\right] d t+\left[\begin{array}{cc}
\Sigma_{S S}(\cdot) & \Sigma_{S Y}(\cdot) \\
0 & \Sigma_{Y Y}(\cdot)
\end{array}\right]\left[\begin{array}{c}
d W_{0 t}^{Q} \\
d W_{t}^{Q}
\end{array}\right]
$$

with $\Sigma_{Y Y}(\cdot)$ full rank. Then, a consistent forward variance curve $G \in C^{2,1}:(y, \tau) \in \mathcal{Y} \times \mathbb{R}_{+} \rightarrow \mathbb{R}_{+}$ $\left(\mathcal{Y} \subset \mathbb{R}_{+}^{d}\right)$ satisfies

$$
\frac{\partial G(y, \tau)}{\partial \tau}=\sum_{i=1}^{d} \frac{\partial G(y, \tau)}{\partial y_{i}} \mu_{Y^{\tau_{i}}}^{Q}(y)+\frac{1}{2} \sum_{i, j=1}^{d} \frac{\partial^{2} G(y, \tau)}{\partial y_{i} \partial y_{j}}\left[\Sigma_{Y} \Sigma_{Y}^{\prime}\right]^{(i, j)}(y)
$$

with d integral boundary conditions

$$
\frac{1}{\tau_{j}} \int_{0}^{\tau_{j}} G\left(y_{j}, y_{-j}, \tau\right) d \tau=y_{j} \text { for all } y_{-j} \in \mathcal{Y}_{-j}
$$

where $\left[\Sigma_{Y} \Sigma_{Y}^{\prime}\right]^{(i, j)}$ denotes the $(i, j)^{\prime}$ th element of $\Sigma_{Y} \Sigma_{Y}^{\prime}$, and satisfying

$$
\mu_{S}^{Q}(\cdot)=-\frac{G_{t}(y, 0)}{2} .
$$

Proposition 1 can be interpreted as an inverse problem: variance swap rates (directly observable quantities) can be seen as families of conditional expectations, indexed by time-to-maturity, over the volatility dynamics (the quantity of interest). Notice that having a finite number of sources of uncertainty in the volatility dynamics is the key assumption that enables us to fully recover the dynamics of the spot volatility conditional on observation of at least the same number of different time-to-maturity variance swap rates. This feature together with the Markovian structure of the model allows us to write the spot variance in terms of the observed rates.

This result is related to Bühler (2006), who characterized consistent variance curve functionals in terms of finite dimensional Markov latent processes. Going further, we exploit the link between variance swap rates and volatility dynamics to write the model in terms of observable factors. By doing so, we obtain additional restrictions in terms of integral boundary conditions which explicitly equate predicted prices with observed prices. The latter ensure that drift and diffusion functions are consistently defined. More specifically, the integral boundary conditions restrict the parameter space of the drift and diffusion

\footnotetext{
${ }^{5}$ In the same way that the term structure of interest rates can be characterized by the instantaneous forward curve, our objective is to determine the term structure of expected integrated variance by the forward variance curve.
} 
functions of variance swap rates further when $d$ increases, i.e., when additional variance swap rates and sources of uncertainty are added to the model.

Finally, positivity constraints on the variance curve need to be imposed in order to obtain properly defined forward variances. This in turn restricts the domain of admissible variance swap rates. This issue will be discussed in more detail when dealing with specific examples.

\subsection{Characterization of affine variance curves}

In Proposition 1, we characterized variance curves in terms of variance swap rates under absence of arbitrage. Interestingly, the result is independent of the specific functional forms of the drift and diffusion functions of variance swap rates, even though it restricts drift and diffusion parameters through the integral boundary conditions. Without further assumptions, however, analytical expressions for every possible consistent model are not available in general. The class of consistent models is simply too large and needs to be further restricted in order to be made explicit. We now derive analytical expressions for the variance curves, $G$, under the following three assumptions:

Assumption 1. The variance curve $G$ is affine in $Y^{d}$.

Assumption 2. The drifts of the variance swap rates are affine- $Q, \mu_{Y_{i}}^{Q}(y)=\alpha_{0 i}+\sum_{j=1}^{d} \alpha_{j i} y_{j}$ for $i=1, \ldots, d$.

Assumption 3. $Y^{d}$ is stationary.

Assumptions 1 and 2 have a natural interpretation: Variance swap rates can be written as linear functions of the underlying unobserved volatility factors as long as the drift functions of the volatility factors are affine in the volatility factors (see e.g. Egloff et al. (2010)). In this particular case, volatility factors are variance swap rates. Assumption 3 rules out cases of explosive volatility dynamics.

We next show the existence of a unique affine forward variance curve and find the restrictions on the dynamics of $Y^{d}$ that prevent arbitrage opportunities. In order to solve (2.6) we make use of the "method of characteristics", which consists of determining curves (called "characteristic curves") along which the partial differential equation (PDE) becomes an ordinary differential equation (ODE). Once the solution of the ODE is found, it is transformed back into a solution of the original PDE. ${ }^{6}$

We can now state our main result in the following form:

Proposition 2. Under assumptions 1-3:

1. There is a unique affine function $G(y, \tau)$ that consistently represents the forward variance curves, which is given by

$$
G(y, \tau)=\left(1-\sum_{j=1}^{d} \xi_{j}\left(\tau, \lambda_{1}, \ldots, \lambda_{d}\right)\right) \bar{\alpha}+\sum_{j=1}^{d} \xi_{j}\left(\tau, \lambda_{1}, \ldots, \lambda_{d}\right) \cdot y_{j}
$$

\footnotetext{
${ }^{6}$ We could have adopted another strategy in which we postulate an affine $G$ and then obtain conditions on the coefficients (parametrised by $\tau$ ) so that the PDE is satisfied along with the boundary conditions.
} 
where the functions $\xi_{j}$ are such that the PDE and the boundary conditions are satisfied.

2. There are $d+1$ free parameters $\left(\alpha,-\lambda^{\prime}\right)^{\prime} \in \mathbb{R}_{+}^{d+1}$ that fully characterize the $(d+1) \times d$ coefficients of $\mu_{Y_{i}}^{Q}(y)$. $d$ of them which are denoted by $\lambda_{1}, \ldots, \lambda_{d}$ can be interpreted as speed of reversion parameters of the system and fully determine $\alpha_{j i}$ for $i, j=1, \ldots, d$ while the remaining one, $\bar{\alpha}$, which is the steady state level of the system, and given $\alpha_{j i}$ for all $i, j: j \neq 0$, determine $\alpha_{0 i}$ for $i=1, \ldots, d$.

\section{Moreover}

$$
\left[\Sigma_{S S}(\cdot), \Sigma_{S Y}(\cdot)\right]^{\prime}\left[\Sigma_{S S}(\cdot), \Sigma_{S Y}^{\prime}(\cdot)\right]=G(y, 0),
$$

and there are no restrictions on $\Sigma_{Y Y}(\cdot)$.

Additionally, we need to impose that the model is well defined in the sense of having a term structure of forward variance that is non-negative, i.e. $G(y, \tau) \geq 0$ for all $\tau \geq 0$. To do so, we identify the region in the $y$-hyperplane for which $G(y, \tau) \geq 0$ for all $\tau \geq 0$. For that purpose, pick a value $y \in \mathbb{R}_{+}^{d}$ such that $G\left(y, \tau^{\prime}\right)=0$ for a given $\tau^{\prime} \geq 0 . G\left(y, \tau^{\prime}\right)=0$ implicitly defines a straight line in the $y$-space such that in any neighborhood of $s^{\prime}$ we find positive and negative forward variances. To determine the envelope, i.e. the set of $y$ 's as a function of $s$ such that $G(y, \tau)$ remains zero for small variations of $s$, we complete the system with $\partial G(y, s) / \partial \tau=0$ by solving:

$$
\left\{\begin{array}{c}
\left(1-\sum_{j=1}^{d} \xi_{j}\right) \bar{\alpha}+\sum_{j=1}^{d} \xi_{j} \cdot y_{j}=0 \\
\left(1-\sum_{j=1}^{d} \partial \xi_{j} / \partial \tau\right) \bar{\alpha}+\sum_{j=1}^{d} \partial \xi_{j} / \partial \tau \cdot y_{j}=0 .
\end{array}\right.
$$

The second equation guarantees that we remain on the frontier as we move in the $y$-space. Once this bound is found, it remains to determine the subregion in which $G(y, \tau)=0$ holds. But given the linearity of $G(\cdot)$ in $y$, this becomes straightforward since we can easily define the region $\mathcal{Y} \in \mathbb{R}_{+}^{d}$ for which the condition is satisfied given the fundamental parameters $\lambda_{1}, \ldots, \lambda_{d}$ and $\bar{\alpha}$, as will be shown in the next subsection.

\subsection{Examples of consistent models}

\subsubsection{The 1-VS family}

We start with the simplest case in which there are just two sources of uncertainty in the economy, one specific to variance swap rates and one common to both variance swap rates and the stock (i.e. $d=1$ ). This model nests several stochastic volatility models already considered in the literature, such as e.g. Heston (1993) and Jones (2003), which in our framework correspond to specific choices of the diffusion function.

By applying Proposition 2, we obtain the following characterization of consistent models: 
Corollary 1. Let $\mu_{Y_{i}}^{Q}\left(y_{1}\right)=\alpha_{01}+\alpha_{11} y$, then variance curve models consistent with the dynamics implied by $\mu_{Y_{i}}^{Q}\left(y_{1}\right)$ are given by

$$
G\left(y_{1}, \tau\right)=\left[1-\xi_{1}\left(\lambda_{1}, \tau\right)\right] \bar{\alpha}+\xi_{1}\left(\lambda_{1}, \tau\right) y_{1}
$$

with

$$
\xi_{1}\left(\lambda_{1}, \tau\right)=\frac{\lambda_{1} \tau_{1}}{1-e^{-\lambda_{1} \tau_{1}}} e^{-\lambda_{1} \tau}
$$

where $\lambda_{1}$ and $\bar{\alpha}$ denote the speed of mean reversion and the steady state level of the dynamic system, respectively. Diffusion functions, apart from (2.10), are unrestricted while drift coefficients are given by $\alpha_{01}\left(\lambda_{1}, \bar{\alpha}\right)=\lambda_{1} / \bar{\alpha}$ and $\alpha_{11}\left(\lambda_{1}\right)=\lambda_{1}$.

Some cross-sectional properties of the linearly mean-reverting one-factor variance curve model (1VS henceforth) can be extracted from the previous definition. First, one-factor stochastic volatility models predict a lower variation in variance swap rates at longer maturities. This matches empirical findings, as for values of the mean reversion speed reported in the recent literature on option pricing using short time-to-maturity option data (e.g. Eraker (2004) and Aït-Sahalia and Kimmel (2007)), the model predicts a much lower (unconditional) variance than the one found in variance swap rates with maturities longer than 6 months. Second, all points in the implied variance term structure become perfectly correlated since the forward variance curve is deterministic conditional on one observed price. Third, this specification only allows - at each point in time- a monotonically upward or downward sloping term structure of forward variances, depending on whether the current spot variance is below or above its long-run level. This is in contrast with the fixed income literature, where linear models with one-factor structure can accommodate hump shaped forward interest rates curves (see e.g. Cox et al. (1985)). At the core of this difference is the fact that variance swap rates are linear in their payoffs.

There are at least two ways of generalizing this model: (i) introducing nonlinearities in the variance curve function $G$, and (ii) allowing for more than one factor as the driving force of volatility.

Extending the model to allow for nonlinearities might be achieved by allowing for non-linear drifts in the variance swap rates or assuming that $G$ is non-linear, hence letting $\partial^{2} G / \partial y_{1}^{2} \neq 0$. However, this comes at a nontrivial cost in terms of numerical tractability, as well as a need of additional assumptions on the functional form of the diffusion function of variance swap rates. This is a straightforward implication from Proposition 1, where the forward variance curve is characterized in terms of (2.6). In general, closed-form solutions would not be available under this setting, making, for instance, derivative pricing significantly more costly.

The second direction, in which several sources of uncertainty are allowed to drive the volatility while keeping its affine structure, is substantially more tractable and practical. First, by retaining the affine structure of the model, we can obtain in a straightforward manner option pricing formulae by using the transform analysis of Duffie et al. (2000), provided one also assumes affine diffusion functions. Second, since the model can be solved analytically without the need of specifying the diffusion functions of variance swap rates, model comparisons become easier both in and out of sample. This is therefore the route we pursue in the next subsection. 


\subsubsection{The 2-VS family}

We now consider the case where there are two sources of uncertainty associated with stochastic volatility in the economy. Applying Proposition 2 allows us to characterize the linearly mean-reverting two-factor variance curve consistent model (2-VS henceforth) as follows:

Corollary 2. Let

$$
\mu_{Y_{1}}^{Q}\left(y_{1}, y_{2}\right)=\alpha_{01}+\alpha_{11} y_{1}+\alpha_{21} y_{2}, \quad \text { and } \mu_{Y_{2}}^{Q}\left(y_{1}, y_{2}\right)=\alpha_{02}+\alpha_{12} y_{1}+\alpha_{22} y_{2}
$$

then variance curve models consistent with the dynamics implied by $\mu_{Y_{i}}^{Q}\left(y_{1}, y_{2}\right)$ for $i=1,2$ are given by

$$
G\left(y_{1}, y_{2}, \tau\right)=\left[1-\xi_{1}\left(\lambda_{1}, \lambda_{2}, \tau\right)-\xi_{2}\left(\lambda_{1}, \lambda_{2}, \tau\right)\right] \bar{\alpha}+\xi_{1}\left(\lambda_{1}, \lambda_{2}, \tau\right) y_{1}+\xi_{2}\left(\lambda_{1}, \lambda_{2}, \tau\right) y_{2}
$$

where for $j=1,2$ and $-j=2,1$, respectively,

$$
\xi_{j}\left(\lambda_{1}, \lambda_{2}, \tau\right)=\frac{\tau_{-j}\left[\lambda_{-j}\left(e^{\lambda_{j} \tau_{j}}-1\right) e^{\lambda_{j} \tau+\lambda_{-j} \tau_{j}}-\lambda_{j}\left(e^{-\lambda_{-j} \tau_{j}}-1\right) e^{\lambda_{-j} \tau+\lambda_{j} \tau_{j}}\right]}{\Xi\left(\lambda_{1}, \lambda_{2}, \tau_{1}, \tau_{2}\right)} e^{\left(\lambda_{1}+\lambda_{2}\right)\left(\tau_{-j}-\tau\right)}
$$

with

$$
\begin{aligned}
\Xi\left(\lambda_{1}, \lambda_{2}, \tau_{1}, \tau_{2}\right) & =e^{\tau_{1}\left(\lambda_{1}+\lambda_{2}\right)}\left(e^{\lambda_{2} \tau_{2}}-e^{\lambda_{1} \tau_{2}}\right)+e^{\lambda_{2} \tau_{1}+\lambda_{1} \tau_{2}} \\
& -e^{\lambda_{1} \tau_{1}+\lambda_{2} \tau_{2}}+e^{\lambda_{1} \tau_{1}+\tau_{2}\left(\lambda_{1}+\lambda_{2}\right)}-e^{\lambda_{2} \tau_{1}+\tau_{2}\left(\lambda_{1}+\lambda_{2}\right)}
\end{aligned}
$$

and $\lambda_{1}$ and $\lambda_{2}$ denoting the speeds of mean reversion, and the steady state level of the dynamic system being denoted by $\bar{\alpha}$. Diffusion functions, apart from (2.10), are unrestricted while drift coefficients for $j=1,2$ and $-j=2,1$ are given by

$$
\begin{gathered}
\alpha_{j,-j}=-\frac{\tau_{j}\left(\lambda_{j}-\lambda_{-j}\right)\left(e^{\lambda_{j} \tau_{-j}}-1\right)\left(e^{\lambda_{-j} \tau_{-j}}-1\right)}{\tau_{-j}\left(e^{\lambda_{j} \tau_{j}}-e^{\lambda_{-j} \tau_{j}}+e^{\lambda_{-j} \tau_{j}+\lambda_{j} \tau_{-j}}-e^{\lambda_{j} \tau_{j}+\lambda_{-j} \tau_{-j}}-e^{\lambda_{j} \tau_{-j}}+e^{\lambda_{-j} \tau_{-j}}\right)} \\
\alpha_{j, j}=\frac{\lambda_{j}\left(1-e^{\lambda_{j} \tau_{j}}\right)\left(e^{\lambda_{-j} \tau_{-j}}-1\right)+\lambda_{-j}\left(e^{\lambda_{-j} \tau_{j}}-1\right)\left(e^{\lambda_{j} \tau_{-j}}-1\right)}{e^{\lambda_{j} \tau_{j}}-e^{\lambda_{-j} \tau_{j}}+e^{\lambda_{-j} \tau_{j}+\lambda_{j} \tau_{-j}}-e^{\lambda_{j} \tau_{j}+\lambda_{-j} \tau_{-j}}-e^{\lambda_{j} \tau_{-j}}+e^{\lambda_{-j} \tau_{-j}}}
\end{gathered}
$$

and

$$
\begin{aligned}
\alpha_{0, j} & =\frac{\lambda_{j}\left(1-e^{\lambda_{j} \tau_{j}}\right)\left(1-e^{\lambda_{-j} \tau_{-j}}\right)-\lambda_{-j}\left(e^{\lambda_{-j} \tau_{j}}-1\right)\left(e^{\lambda_{j} \tau_{-j}}-1\right)}{e^{\lambda_{j} \tau_{j}}-e^{\lambda_{-j} \tau_{j}}+e^{\lambda_{-j} \tau_{j}+\lambda_{j} \tau_{-j}}-e^{\lambda_{j} \tau_{j}+\lambda_{-j} \tau_{-j}}-e^{\lambda_{j} \tau_{-j}}+e^{\lambda_{-j} \tau_{-j}}} \bar{\alpha} \\
& +\frac{\tau_{-j}}{\tau_{j}} \frac{\left(\lambda_{-j}-\lambda_{j}\right)\left(e^{\lambda_{j} \tau_{j}}-1\right)\left(e^{\lambda_{-j} \tau_{j}}-1\right)}{e^{\lambda_{j} \tau_{j}}-e^{\lambda_{-j} \tau_{j}}+e^{\lambda_{-j} \tau_{j}+\lambda_{j} \tau_{-j}}-e^{\lambda_{j} \tau_{j}+\lambda_{-j} \tau_{-j}}-e^{\lambda_{j} \tau_{-j}}+e^{\lambda_{-j} \tau_{-j}}} \bar{\alpha}
\end{aligned}
$$

These types of models allow for a much richer term structure of forward variance than the 1-VS ones. In particular, they allow for flexible term structures of volatilities at intermediate maturities. Figure 1 reports forward volatility curves for a sample of days reflecting different market expectations on volatility of the S\&P 500. Parameter values used to construct the curves are taken from Section 3; specifically, they correspond to the first column in Table 4. For instance, during quiet periods, such as March 1996 or May 2006, volatility curves are typically flat at a level below 20\%, or slightly increasing 
with a small slope. By contrast, during periods of turmoil such as the Asian crisis, such as in the months following the speculative attack against Thailand's currency, in August 1997, the fact that the market was anticipating an increase in volatility over the following months is reflected in the hump-shaped curve of forward volatility. Even more noticeable is the hump-shape the forward volatility curve presents in the aftermath of the dot-com bubble burst, starting in March 2000. The forward volatility curve is different during the weeks following 9/11: the curve signals anticipations of a return of volatility to normal levels in the following months.

Regarding the positivity constraints described in Section 2.3, Figure 2 depicts the feasible regions $\mathcal{Y}$ for different values of the fundamental parameters. In the figure, red (solid) lines denote the restriction for the spot variance to be non-negative while black (dashed) curves represent the limits that prevent violations of this condition for the forward variances for $s>0$.

\section{Econometric implementation}

Our aim is now to provide all necessary elements to estimate affine market-based stochastic volatility models to the data. In brief, this requires specifying the full dynamics of the model under the objective measure $P$ as well as the estimation method. In the first part of Section 3.1, we complete the description of the model under the pricing measure $Q$, given the invariance of our main result to diffusion specifications. We then write the dynamics under $P$ by introducing some parsimonious market prices of risk specifications which determine the change from $Q$ to $P$ dynamics. In Section 3.2, we describe the estimation procedure, and finally, in Section 3.3 we deal with situations in which the cross-section of variance swap rates observed in the data is richer than the theoretical sources of uncertainty in the model.

For expositional purposes, we restrict the discussion to the consistent 1-VS and 2-VS models, as derived in Section 2.4. Generalization of the econometric implementation to a higher number of sources of uncertainty in the economy beyond that is straightforward.

\subsection{Dynamics under the objective measure}

\subsubsection{The diffusion matrix}

As we saw above, the arbitrage-free characterization of volatility dynamics is independent of diffusion specification in the affine-drift context. Hence, at this point all we need to complete the dynamics under $Q$ is a specification of the diffusion matrix $\Sigma_{t}$ which we can set freely. We choose the following parametrizations in order to nest some of the specifications proposed in the literature:

$$
\Sigma_{t}=\sigma_{t}\left(\begin{array}{cc}
\sqrt{1-\gamma_{1}^{2}} & \gamma_{1} \\
0 & \sigma_{t}^{\beta-1} \gamma_{11}
\end{array}\right), \text { with } \sigma_{t}=\sqrt{G\left(Y_{t}^{\tau_{1}}, 0\right)}
$$


for the 1-VS and, for the 2-VS model,

$$
\Sigma_{t}=\sigma_{t}\left(\begin{array}{ccc}
\sqrt{1-\gamma_{1}^{2}-\gamma_{2}^{2}} & \gamma_{1} & \gamma_{2} \\
0 & \sigma_{t}^{\beta-1} \gamma_{11} & \sigma_{t}^{\beta-1} \gamma_{12} \\
0 & 0 & \sigma_{t}^{\beta-1} \gamma_{22}
\end{array}\right) \text {, with } \sigma_{t}=\sqrt{G\left(Y_{t}^{\tau_{1}}, Y_{t}^{\tau_{2}}, 0\right)},
$$

where the expressions for $G\left(Y_{t}^{\tau_{1}}, 0\right)$ and $G\left(Y_{t}^{\tau_{1}}, Y_{t}^{\tau_{2}}, 0\right)$ are given in Corollaries 1 and 2, respectively, and $\Sigma$ 's satisfy (2.10). For identification purposes we restrict the $\gamma_{i i}$ 's to be positive. ${ }^{7}$ Here $\beta$ is the elasticity of volatility of the variance swap rates, which for simplicity, we assume to be the same under different time-to-maturities, while $\gamma_{i j}$ 's define the volatility and correlation structure. Notice also that our definition of $\Sigma_{t}$ is well suited to capture the leverage effect, namely the likely negative correlation between shocks from $W_{0}$ and the remaining components of $W$ and shock to the underlying asset log-returns.

\subsubsection{Market prices of risk}

Bates (2000), Pan (2002) and Aït-Sahalia and Kimmel (2007) among others find that market prices of variance risks are negative and economically large under alternative specifications. For that reason, we do not only model the risk premium associated to $W_{0}$ but also allow the parameterization to capture how the market differentially prices volatility factors. To do so, we consider one market price of risk for each source of uncertainty. For instance, for the case of $\mathbf{W}=\left(W_{0}, W_{1}, W_{2}\right)^{\prime}$ we set

$$
\Lambda_{t}=\sigma_{t}^{\delta}\left(\begin{array}{ccc}
\nu_{0} & \nu_{1} & \nu_{2}
\end{array}\right)^{\prime}
$$

where $\sigma_{t}=\sqrt{G\left(Y_{t}^{\tau_{1}}, Y_{t}^{\tau_{2}}, 0\right)}$ is the stochastic component that drives the risk premia in the model and $\nu$ 's are parameters to be estimated. When $\delta$ is different from zero, risk premia depend on the volatility level. This is an acceptable specification for market prices of risk, since it is associated with fundamental sources of risk and not prices. In other words, if we change the time-to-maturity of the variance swap rate used for estimation, we still obtain the same market prices of risk in the econometric model.

\subsubsection{P-dynamics}

Under $P$, the observed variables of the 2-VS model evolve according to

$$
\begin{aligned}
d \log S_{t} & =\mu_{S}^{P}\left(Y_{t}^{\tau_{1}}, Y_{t}^{\tau_{2}}\right) d t+\sigma_{t}\left(\sqrt{1-\gamma_{1}^{2}-\gamma_{2}^{2}} d W_{0 t}^{P}+\gamma_{1} d W_{1 t}^{P}+\gamma_{2} d W_{2 t}^{P}\right) \\
d Y_{t}^{\tau_{1}} & =\mu_{Y_{1}}^{P}\left(Y_{t}^{\tau_{1}}, Y_{t}^{\tau_{2}}\right) d t+\sigma_{t}^{\beta}\left(\gamma_{11} d W_{1 t}^{P}+\gamma_{12} d W_{2 t}^{P}\right) \\
d Y_{t}^{\tau_{2}} & =\mu_{Y_{2}}^{P}\left(Y_{t}^{\tau_{1}}, Y_{t}^{\tau_{2}}\right) d t+\sigma_{t}^{\beta} \gamma_{22} d W_{2 t}^{P}
\end{aligned}
$$

\footnotetext{
${ }^{7}$ This assumption is without loss of generality given that our ultimate parameter of interest is the covariance structure, which is actually identified in the model.
} 
where

$$
\begin{aligned}
& \mu_{S}^{P}\left(Y_{t}^{\tau_{1}}, Y_{t}^{\tau_{2}}\right)=-\frac{1}{2} \sigma_{t}^{2}+\sigma_{t}^{\delta+1}\left(\nu_{0} \sqrt{1-\gamma_{1}^{2}-\gamma_{2}^{2}}+\nu_{1} \gamma_{1}+\nu_{2} \gamma_{2}\right) \\
& \mu_{Y_{1}}^{P}\left(Y_{t}^{\tau_{1}}, Y_{t}^{\tau_{2}}\right)=\mu_{Y_{1}}^{Q}\left(Y_{t}^{\tau_{1}}, Y_{t}^{\tau_{2}}\right)+\sigma_{t}^{\delta+\beta}\left(\nu_{1} \gamma_{11}+\nu_{2} \gamma_{22}\right) \\
& \mu_{Y_{2}}^{P}\left(Y_{t}^{\tau_{1}}, Y_{t}^{\tau_{2}}\right)=\mu_{Y_{2}}^{Q}\left(Y_{t}^{\tau_{1}}, Y_{t}^{\tau_{2}}\right)+\sigma_{t}^{\delta+\beta} \nu_{2} \gamma_{22} .
\end{aligned}
$$

This parameterization nests important special cases which have been extensively analyzed in the previous literature on empirical option pricing (e.g. Aït-Sahalia and Kimmel (2007)): (i) $\beta=1$, then the variance swap rates are square-root type processes in terms of the spot volatility; moreover, if $\delta=1$, the drift functions of the variance swap rates are affine- $P$ in the variance swap rates; (ii) $\beta=2$, then the variance swap rates are GARCH type processes in terms of the spot volatility; if in addition $\delta=0$, again the drift functions of the variance swap rates are affine- $P$ in the variance swap rates. More generally when $\delta+\beta=2, \mu^{P}($.$) 's are affine- P$ in the variance swap rates.

Market prices of risk shape the term structure of the unconditional level (or long-run level) of variance swap rates. Under $Q$, the term-structure is flat and equal to $\bar{\alpha}$ as a consequence of the fact that $\mu^{Q}($.$) is affine and hence, the long-run level of the rates is common for all maturities. However,$ under $P$, market prices of risk may add nonlinearities in the drift functions of variance swap rates, thereby delivering richer possible term structures. The long-run level of the variance swap rates can then be found as $y^{*}$ such that $\mu^{P}\left(y^{*}\right)=0$ i.e. the long run variance curve as the steady state of the dynamic system.

In the situations described above, in which drifts are affine under both measures, the intercepts of the drift functions are the ones that determine the long run level of variance of the model. Specifically, focusing on the 1-VS model, if variance risk is negative, then the term structure is also flat, but with a long-run level of variance that is lower under the objective measure. In the more general case of non-affine $P$-drifts, i.e. when $\delta+\beta \neq 2$, if we take again the 1 -VS $Q$-affine family, the long-run variance swap term structure will be upward sloping if the market price of variance risk $\nu_{1}$ is negative, provided $\beta+\delta>2$.

\subsection{Closed-form likelihood expansions}

We now expand the $\log$-likelihood function, $l_{X}:=\log p_{X}$, of the model under $P$, using Aït-Sahalia (2008)'s closed form method. The expansion takes the form of a power series with additional leading terms in $\Delta$, the time interval separating observations,

$$
l_{X}\left(\Delta, x \mid x_{0} ; \phi\right)=-\frac{d+1}{2} \log (2 \pi \Delta)-D_{v}(x ; \phi)+\frac{C_{X}^{(-1)}\left(x \mid x_{0} ; \phi\right)}{\Delta}+\sum_{k=0}^{J} C_{X}^{(k)}\left(x \mid x_{0} ; \phi\right) \frac{\Delta^{k}}{k !},
$$

where

$$
D_{v}(x ; \phi)=\frac{1}{2} \log \{\operatorname{det}[v(x ; \phi)]\}, \text { with } v(x ; \phi)=\Sigma_{X}(x ; \phi) \Sigma_{X}^{\prime}(x ; \phi),
$$


and $\phi$ denotes the extended parameter vector of the model. The series can be calculated up to arbitrary order $J$. To be determined are the coefficients $C_{X}^{(k)}$ corresponding to each $\Delta^{k}, k=-1,0, \ldots, J$. The method provides a Taylor series in $\left(x-x_{0}\right)$ for each coefficient $C_{X}^{(k)}$, at order $j_{k}$, which is fully explicit. Such an expansion will be denoted by $C_{X}^{\left(j_{k}, k\right)}$, and is taken at order $j_{k}=2(J-k)$.

The resulting expansion is then

$$
\tilde{l}_{X}\left(\Delta, x \mid x_{0} ; \phi\right)=-\frac{d+1}{2} \log (2 \pi \Delta)-D_{v}(x ; \phi)+\frac{C_{X}^{\left(j_{-1},-1\right)}\left(x \mid x_{0} ; \phi\right)}{\Delta}+\sum_{k=0}^{J} C_{X}^{\left(j_{k}, k\right)}\left(x \mid x_{0} ; \phi\right) \frac{\Delta^{k}}{k !} .
$$

While we implement this for the models 1 -VS and 2 -VS, ${ }^{8}$ the coefficients $C_{X}^{\left(j_{k}, k\right)}$ can be obtained in closed form for arbitrary specifications of the dynamics of the state vector $X_{t}$ by solving a system of linear equations.

More importantly, given the non-linear nature in which the parameters enter in the model, (3.5) is useful to give us insights about the precision we should expect for the different parameters entering in the models. Again focusing on the 1-VS family, we distinguish between the structural parameter vector $\theta=\left(\bar{\alpha}, \lambda_{1}, \nu_{0}, \nu_{1}, \gamma_{1}, \gamma_{11}, \beta, \delta\right)^{\prime}$ and the extended parameter vector which directly enters into the likelihood whose evaluation can be directly computed from Proposition 2, that is

$$
\phi(\theta)=\left(\alpha_{01}\left(\bar{\alpha}, \lambda_{1}\right), \alpha_{11}\left(\bar{\alpha}, \lambda_{1}\right), \nu_{0}, \nu_{1}, \xi_{1}\left(\bar{\alpha}, \lambda_{1}\right), \gamma_{1}, \gamma_{11}, \beta, \delta\right)^{\prime}
$$

Using the extended parameter vector simplifies the computations of the likelihood function, since the non-linear relationship between $\left(\bar{\alpha}, \lambda\right.$ 's) and ( $\alpha$ 's, $\xi^{\prime}$ 's) can be treated separately using the mapping $\phi(\theta)$. Notice that all but $\nu$ 's and $\delta$ enter in both drift and diffusion equations.

Only the diffusion coefficients enter into $C_{X}^{\left(j_{-1},-1\right)}\left(x \mid x_{0} ; \phi\right)$, which is the leading term, so we should expect precise estimates of $\gamma$ 's and $\beta$, as we sample more frequently, but also for $\lambda$ 's and $\bar{\alpha}$, since they also enter into $\Sigma$ through $G\left(y_{1}, y_{2}, 0\right)$. As for $\nu$ 's and $\delta$, which are only identified through the drift functions, the quality of the estimates of those parameters will typically depend primarily on the length of the sample, rather than on the sampling frequency. The actual performance of the estimator of the different groups of parameters in small samples is described in Appendix 2.

\subsection{Additional variance swap rates}

The assumption that there is a fix number of sources of uncertainty that drive volatility allows us to determine the price of any time-to-maturity variance swap using the same number of different time to maturity reference rates as inputs. If observations on more variance swap rates are available, we can exploit the resulting overidentification in order to improve the precision of parameter estimates. In particular, this source of cross-sectional information might prove useful to better estimate those parameters whose main role is through the variance curve, namely, the drift parameters under $Q$, which are the ones that enter in a more non-linear manner within the drift coefficients.

\footnotetext{
${ }^{8}$ The resulting coefficients $C_{X}^{\left(j_{k}, k\right)}$ for the 1-VS and 2-VS models are available in computer form upon request.
} 
Assume we have observations on $N>d$ different time-to-maturity variance swaps rates, as in the fixed income literature where more bonds yields are observed than there are factors in the term structure model. Since there are only $d+1$ sources of risk and we observe $N+1$ processes, we assume that $N-d$ processes are observed with error: for instance, one could think of variance swap rates with longer maturities as the natural candidates since those tend to be less liquid.

From the coefficients of the model and the value of $Y^{\tau_{1}}, \ldots, Y^{\tau_{d}}$, we can calculate the implied value of a variance swap with maturity $\tau_{n}, Y^{\tau_{n}}$, at every $t$ by

$$
\hat{Y}_{t}^{\tau_{n}}\left(Y_{t}^{\tau_{1}}, \ldots, Y_{t}^{\tau_{d}}, \bar{\alpha}, \lambda_{1}, \ldots, \lambda_{d} ; \tau_{n}\right)=\frac{1}{\tau_{n}} \int_{0}^{\tau_{n}} G\left(Y_{t}^{\tau_{1}}, \ldots, Y_{t}^{\tau_{d}}, \bar{\alpha}, \lambda_{1}, \ldots, \lambda_{d} ; s\right) d s .
$$

If we specialize to the 1 -VS model and let $Y^{\tau}$ be a variance swap rate with $\tau \neq \tau_{1}$ then

$$
\hat{Y}^{\tau}\left(Y_{t}^{\tau_{1}}, \bar{\alpha}, \lambda_{1}\right)=\bar{\alpha}+\frac{\tau_{1}}{\tau} \frac{1-e^{-\lambda_{1} \tau}}{1-e^{-\lambda_{1} \tau_{1}}}\left(Y_{t}^{\tau_{1}}-\bar{\alpha}\right) .
$$

Then, additive (or multiplicative) pricing errors can be defined as the difference (or difference of logs) between the theoretical values and the observed values. For instance, in the case of additive pricing errors, these errors are simply defined as the difference between these implied values and the observed values i.e.

$$
\left[\begin{array}{c}
\varepsilon_{t}^{\tau_{d+1}}\left(Y_{t}^{\tau_{1}}, \ldots, Y_{t}^{\tau_{d}}, \theta ; \tau_{d+1}\right) \\
\vdots \\
\varepsilon_{t}^{\tau_{N}}\left(Y_{t}^{\tau_{1}}, \ldots, Y_{t}^{\tau_{d}}, \theta ; \tau_{N}\right)
\end{array}\right]=\left[\begin{array}{c}
Y_{t}^{\tau_{d+1}} \\
\vdots \\
Y_{t}^{\tau_{N}}
\end{array}\right]-\left[\begin{array}{c}
\hat{Y}_{t}^{\tau_{d+1}}\left(Y_{t}^{\tau_{1}}, \ldots, Y_{t}^{\tau_{d}}, \bar{\alpha}, \lambda_{1}, \ldots, \lambda_{d} ; \tau_{d+1}\right) \\
\vdots \\
\hat{Y}_{t}^{\tau_{N}}\left(Y_{t}^{\tau_{1}}, \ldots, Y_{t}^{\tau_{d}}, \bar{\alpha}, \lambda_{1}, \ldots, \lambda_{d} ; \tau_{N}\right)
\end{array}\right]
$$

We assume that pricing errors are Gaussian with zero mean and constant variance, and that they are independent across time and maturity, and also independent of $\mathbf{W}$. Because of the independence assumption, the joint log-likelihood of the panel of observed variance swaps is simply the sum over the observations of

$$
l_{X}\left(\Delta,\left(\log S_{t}, Y_{t}^{\tau_{1}}, \ldots, Y_{t}^{\tau_{d}}\right)^{\prime} \mid\left(\log S_{t-\Delta}, Y_{t-\Delta}^{\tau_{1}}, \ldots, Y_{t-\Delta}^{\tau_{d}}\right)^{\prime} ; \phi(\theta)\right)
$$

so that

$$
l\left(\left(Y_{t}^{\tau_{d+1}}, \ldots, Y_{t}^{\tau_{N}}\right)^{\prime} \mid Y_{t}^{\tau_{1}}, \ldots, Y_{t}^{\tau_{d}} ; \theta, \theta_{E}\right)=\sum_{j=d+1}^{N} \log \frac{1}{\sigma_{j}} \varphi\left(\frac{\varepsilon_{t}^{\tau_{j}}\left(Y_{t}^{\tau_{1}}, \ldots, Y_{t}^{\tau_{d}}, \theta ; \tau_{j}\right)}{\sigma_{j}}\right)
$$

where $\theta_{E}=\left(\sigma_{d+1}^{2}, \ldots, \sigma_{N}^{2}\right)^{\prime}$ and $\varphi($.$) denotes the standard normal probability density function. Equiva-$ lently, the joint likelihood of the panel of all observed variance swap rates is simply the product of the likelihood of the rates observed without error and the likelihood of the observation errors.

\section{Empirical application}

\subsection{The data}

We now estimate the models derived in the previous sections using daily data on the $\mathrm{S} \& \mathrm{P} 500$ index returns and variance swap rates on five different maturities (2, 3, 6, 12 and 24 months) over the period 
January 4, 1996 to March 30, 2007. For the full sample, the number of daily observations is 2,827, excluding weekends and holidays. Data on variance swaps quotes are obtained from Bank of America.

Descriptive statistics for S\&P index returns, VIX, and variance swap rates are in Panel A of Table 1. Two features are noteworthy: first, the unconditional term structure is upward-sloping, suggesting that the market price of variance risk is negative. Second, standard deviations are similar irrespective of the maturity of the contract, which may suggest the presence of a persistent factor in the volatility dynamics. Third, the eigenvalue decomposition of the covariance matrix of variance swap rates in Panel $\mathrm{B}$ of the same table shows that the main principal component explains around $90 \%$ of the total variation in the data, while the first two explain more than $99 \%$. The corresponding eigenvectors suggest that the first eigenvalue is related to level shifts in the variance curve while the second one captures changes in the slope. Interestingly, when compared with the values of Litterman and Scheinkman (1991) for bond returns, we find a simpler structure in the term structure of variances than the corresponding one in bond returns.

\subsection{Taking the models to the data}

Each trading day is considered to be $\Delta=1 / 252$ after the previous day, regardless of the calendar time passed (i.e. weekends and holidays receive no special consideration). Standard errors are calculated by evaluating the sample means of the cross products of the score functions, which in turn have been calculated by numeric differentiation of the log likelihood. Guided by the Monte Carlo experiments reported in Appendix 2, $\delta$ is not estimated and remains fixed to 1 -square-root market prices of risksexcept in Section 4.2.2 below, in which we also consider the case of constant market prices of risks -that is, $\delta=0$ - in order to assess the impact of alternative specifications for the risk premia parameters.

\subsubsection{Fitting some common specifications in the one-factor stochastic volatility literature}

Table 2 reports parameter estimates for the 1-VS model for different specifications of the diffusion function. The first one, Heston-type diffusion $(\beta=1)$, imply affine drift under the objective measure, while the remaining two, GARCH-type diffusion $(\beta=2)$ and CEV-type diffusion do not. Results are grouped in two panels according to the time-to-maturity of the reference variance swap rate. Panel A contains parameter estimates and standard errors using data on the spot and 2-month swap rates, while 2, 6 and 12-month rates are assumed to be observed with error. Panel B does the same but uses the 6-month swap rate as the reference rate.

Parameter estimates in Panel A are somewhat different from values found in recent works based on data from stock returns and/or short dated option prices (Pan (2002), Eraker (2004) and Aït-Sahalia and Kimmel (2007) among others): values of $\bar{\alpha}$ that suggest a moderately high steady-state level of volatility around $27-35 \%$, and a speed of mean reversion smaller than 1 , which is lower than the values that one can find by fitting stochastic volatility models to short time-to-maturity option prices. When the reference maturity is 6 months (Panel B) the pattern of high unconditional level of volatility and 
low mean reversion is even stronger. Interestingly, the parameter values of the drift under $Q$ are quite robust to the specification of the diffusion function, with the exception of the CEV estimates of $\bar{\alpha}$ and $\lambda_{1}$ being lower and higher in absolute value, respectively.

The large uncertainty for the risk premia estimates is perhaps not surprising, given that the sample period is only 12 years long, and that risk premia are typically poorly estimated even in longer samples. These parameters pertain to the drift under the objective measure $P$, and the quality of the estimates of those parameters typically depends only on the length of the sample, and not the sampling frequency. Given the relative novelty of the variance swaps contract and the resulting length of the available data, there is little that can be done to improve the quality of the $\nu$ 's estimates, apart from waiting for more data to accrue. In contrast, diffusion parameters are estimated with high precision since their asymptotics depends on the sampling frequency $(\Delta=1 / 252) .{ }^{9}$ The volatility and risk premia parameters are not directly comparable across columns owing to the differing diffusion specifications. However, when looking to the same column across panels the volatility parameter $\gamma_{11}$ is smaller in the case of the 6-month time-to-maturity variance swap rate. This is a consequence of the fact that in 1-VS models the unconditional volatility of variance swap rates is decreasing in time-to-maturity. Finally, all the specifications suggest strong negative correlation $\gamma_{1}$ between shocks to the stock and the volatility variable. The robustness of this finding across models suggests that stochastic volatility models, pricing and/or estimation methods that rely on the assumption of uncorrelated shocks, such as Hull and White (1987), will be quite unrealistic in this context.

\subsubsection{1-VS versus 2-VS models and market prices of risk specification}

As discussed in Section 2.4.1, one implication of one-factor models is that all points in the implied variance term structure become perfectly correlated. Moreover, they only allow for a monotonically upward or downward sloping term structure of forward variances at a given point in time, depending on whether the current spot variance is below or above its long-run level. The 2-VS family of Section 2.4.2, by contrast, allows for richer term structures. We now compare the ability of these two families of consistent models to fit the data.

In order to assess the models' fit, we consider two sampling periods: (i) the full sample, and (ii) a subsample that starts in October 10, 1997 and ends September 30, 2003 and is characterized by high average volatility in the market, as can be seen in Figure 3. It includes the Asian and Russian financial crises, the LTCM bailout, and the burst of the dot-com bubble. The number of daily observations for the subsample is 1,500, as is the case in the corresponding Monte Carlo design of Appendix 2.

Tables 3 and 4 report parameter estimates and standard errors for the affine specification $(\beta=1)$ of the diffusion matrix for the 1-VS and 2-VS models, respectively. As for the 1-VS model, it should be noted -using the 2-month rate as the reference variance swap rate- that both the speed of mean

\footnotetext{
${ }^{9}$ To take an extreme case, consider an arithmetic or geometric Brownian motion. The volatility can be estimated to an arbitrary degree of precision by sampling frequently enough, but the drift estimate is independent of sampling frequency. The first and last observations provide as good an estimate of the drift as weekly, daily or hourly observations.
} 
reversion and the unconditional level of volatility are unstable if we compare the parameter estimates from the full sample with ones from the subsample. The volatility parameters estimates are larger in the subsample, reflecting the higher average volatility during that period. Similarly, $\bar{\alpha}$ estimates are smaller when the full sample is used. In short, in a 1-VS model world, this 6 year period would be understood as being far from the steady state.

We also estimate the model with constant market prices of risk i.e. $\delta=0$ and report these estimates and standard errors in the last columns of Table 3. The estimates for the majority of the parameters are almost identical to the case of $\delta=1$. This reflects the fact that $\delta$ is weakly identified with this type of data: Only $\nu$ 's estimates change in order to correct the change of scale $\sigma_{t}$ to 1 .

Table 4 contains the results for the 2-VS model. In this case, we need to fix two reference rates, and we use the 6 -month rate in addition to the 2 -month swap rate. In contrast to the 1-VS model, parameter estimates are more robust to the choice of the sampling period. The speed of reversion associated to the low frequency, $\lambda_{1}$, implies that shocks to volatility last for more than one year on average, while the fast mean reverting root, $\lambda_{2}$, suggest that the mean life of this type of shocks is less than 2 months in both samples. The speed of mean-reversion estimated for the 1-VS model, which lies in between the estimates of the mean-reverting roots $\lambda_{1}$ and $\lambda_{2}$ of the 2-VS model, is closer to $\lambda_{1}$. This feature can be associated with the fact that most of the variation in the variance curves correspond to level shifts, as seen from the principal components decomposition of the variance swap rates.

Again, the estimates of the volatility parameters are larger in the subsample reflecting the higher average volatility during that period. As in the 1-VS case, the choice of the functional form of the market prices of risk (i.e. the specific value for $\delta$ ) does not seem to affect the parameters that characterize the dynamics of the variance swap rates.

Risk premia coefficients are not precisely estimated. Still, it is noteworthy that the negative market price estimate on the variance risk is mainly captured through $\nu_{2}$ rather than $\nu_{1}$. This is due to the parameterization of the diffusion matrix (since $W_{2}$ affect both reference rates). This fact captures the evidence that variance swap rates are higher on average than ex-post realized variance, and that shorting variance swap contracts generates positive average returns, observations also made by Bondarenko (2004) and Carr and Wu (2009).

Analyzing the properties of pricing errors allows us to assess, in an informal but intuitive way, the statistical performance of the different models. Under correct specification, pricing errors should be uncorrelated and have small variance. The variance parameters that characterize the pricing errors are reported in the bottom panels of Tables 3 and 4 . We can see that the one-factor model seems to perform poorly relative to the 2-VS model since estimates of $\sigma^{2}$ in the 2-VS models are in most cases remarkably smaller than in the 1-VS model.

Finally, we resort to the model selection criterion based on the likelihood ratio for non-nested models (see Theorem 5.1 in Vuong (1989)) to evaluate the fit of the alternative specifications more rigorously. Specifically, when evaluating strictly non-nested models $A$ and $B$, we compute the test statistic as 
$Z_{A, B}=T^{-1 / 2} L R_{T}\left(\phi_{A}, \phi_{B}\right) / \hat{\omega}_{T}$, which is standard normal if $A$ and $B$ are equivalent, and diverges to $\div \infty(-\infty)$ if model $A(B)$ is better than $B(A) \cdot{ }^{10}$ Using the output from the full sample estimation we find that $Z_{(1 V S, \delta=1),(2 V S, \delta=1)}=-120.05$ and $Z_{(1 V S, \delta=0),(2 V S, \delta=0)}=-126.72$, providing strong support in favor of the 2-VS family. As for the market prices of risk specifications, we find evidence in favor of the time-varying specification for the 2-VS model, while the 1-VS seems to do a better job with constant market prices of risk since $Z_{(1 V S, \delta=1),(1 V S, \delta=0)}=-3.15$ and $Z_{(2 V S, \delta=1),(2 V S, \delta=0)}=11.74{ }^{11}$

This is even more evident if we look at the distribution of the pricing errors estimates for both models. Panel A of Figure 4 presents histograms of the 3-month (top) and 1-year (bottom) rates pricing errors for both 1-VS (left) and 2-VS (right) models using the MLE estimates from data on 2, 3, 6 and 12-month time-to-maturity. Panel B of Figure 4 does the same for 1-month (top) and 2-year (bottom) pricing errors. Intuitively, errors in Panel A can be interpreted as in-sample forecasting errors, while those in Panel B have an out-of-sample interpretation. In both figures, it is clear that the 2-VS outperforms the 1-VS model. The reasonable performance achieved through the inclusion of an extra factor in fitting variance derivatives that are not used for estimation suggests that the 2-VS models could constitute a plausible representation of the data.

\subsubsection{The impact of additional variance swap rates on statistical efficiency}

In the previous subsections, we estimated the models using additional rates - either two or three, depending on the model,-- since they provide a better fit of the relevant parameters, as we have learned from the Monte Carlo simulations. Now, we explicitly compare the fit of the models using different information sets. Panels A and B of Table 5 present parameter estimates for the affine specification of the diffusion matrix for the 1-VS and 2-VS models, respectively.

Not surprisingly, we see that the precision of parameter estimates increases as we add information on different maturities. The main gain arises from increasing the data set in which we base inference from using just the reference rates to using a few additional rates. The precision of drift parameters estimates under $Q$ is increased by exploiting the cross-sectional structure of prices since the variance curve is determined by those parameters only. Along the same line, but at a lower rate, the standard errors of diffusion parameters become smaller as the contribution to the likelihood of different maturities is taken into account. This is not the case, however, with the $P$-parameters that characterize the drift under the physical measure, namely, the market prices of risk $\nu$ 's.

When using data on the six maturities, the mean-life of shocks to the low frequency component of volatility increases (i.e. $\lambda_{1}$ approaches zero), as can be seen from the estimates of $\lambda$ 's. Similarly, the long-run level of variance $\alpha$ increases as we use information from longer maturities. As for the diffusion

${ }^{10}$ The variance of the limiting normal distribution (when the models are equivalent) $\hat{\omega}_{T}^{2}$ is estimated by

$$
\hat{\omega}_{T}^{2}=\frac{1}{T} \sum_{t=1}^{T}\left[\log f_{A}\left(y_{t} ; \phi_{A}\right)-\log f_{B}\left(y_{t} ; \phi_{B}\right)\right]^{2}-\left[\frac{1}{T} \sum_{t=1}^{T} \log f_{A}\left(y_{t} ; \phi_{A}\right)-\log f_{B}\left(y_{t} ; \phi_{B}\right)\right]^{2}
$$

${ }^{11}$ We arrive to the same conclusions when using the subsample period. 
parameters, the vol-vol parameters $\gamma_{11}$ and $\gamma_{22}$, and the correlation parameters $-\gamma_{1}, \gamma_{2}$ and $\gamma_{12}-$ are quite stable.

\section{Conclusions}

We developed in this paper stochastic volatility models based on variance swaps as their primitives, derived the consistent class of models, and proposed and implemented an estimation approach for the resulting models. Using no-arbitrage arguments to characterize families of consistent variance curve models parameterized in terms of observed variance derivatives, we are able to estimate stochastic volatility without relying on latent variables assumptions. By specifying the model in terms of observed quantities, estimation of stochastic volatility models becomes noticeably simpler.

Empirical results for simple versions of the 1-VS and 2-VS models suggest that at least two sources of uncertainty are required in order to get a reasonable description of the dynamics of variance swap rates. The long-run risk that is associated with a near martingale behavior of one of the factors, suggests that agents face large uncertainty about the future level of volatility, and it has an important impact in prices of variance derivatives and risk premia at long horizons.

By contrast, fitting one-factor stochastic volatility models to the term structure of variance derivatives may lead to the following potentially misleading results: (i) low mean reversion of the spot variance, which is not consistent with the observed mean reversion from the analysis of short time-to-maturity derivatives; and (ii) understatement of the long-run risk, the dominant component for explaining the variance risk premia.

Finally, when we compare the fit of the models using different information sets, the main efficiency gains arise from increasing the data set in which we base inference from using just the reference rates to using a few additional rates. Specifically, the precision of drift parameters estimates under the pricing measure $Q$ is increased by exploiting the cross-section of prices. 


\section{References}

Aït-Sahalia, Y., 1996. Testing continuous-time models of the spot interest rate. Review of Financial Studies 9, 385-426.

Aït-Sahalia, Y., 2008. Closed-form likelihood expansions for multivariate diffusions. Annals of Statistics $36,906-937$.

Aït-Sahalia, Y., Amengual, D., Manresa, E., 2014. Market-based estimation of stochastic volatility models. Tech. rep., Princeton University.

Aït-Sahalia, Y., Kimmel, R., 2007. Maximum likelihood estimation of stochastic volatility models. Journal of Financial Economics 83, 413-452.

Albanese, C., Carr, P., Madan, D. B., 1998. Stochastic implied volatility. Tech. rep., Bank of America Securities.

Amengual, D., 2008. The term structure of variance risk premia. Tech. rep., Princeton University.

Bates, D. S., 2000. Post-' 87 crash fears in the S\&P 500 futures option market. Journal of Econometrics 94, 181-238.

Bergomi, L., 2005. Smile dynamics II. RISK 73, 18-67.

Black, F., Scholes, M., 1973. The pricing of options and corporate liabilities. Journal of Political Economy $81,637-654$.

Bollerslev, T., Sizova, N., Tauchen, G. T., 2012. Volatility in equilibrium: Asymmetries and dynamic dependencies. Review of Finance 16, 31-80.

Bondarenko, O., 2004. Market price of variance risk and performance of hedge funds. Tech. rep., University of Illinois at Chicago.

Bühler, H., 2006. Consistent variance curve models. Finance and Stochastics 10, 178-203.

Carr, P., Wu, L., 2009. Variance risk premiums. Review of Financial Studies 22, 1311-1341.

Christoffersen, P., Heston, S., Jacobs, K., 2009. The shape and term structure of the index option smirk: Why multifactor stochastic volatility models work so well. Management Science 55, 1914-1932.

Cont, R., da Fonseca, J., Durrleman, V., 2002. Stochastic models of implied volatility surfaces. Economic Notes 31, 361-377.

Cox, J. C., Ingersoll, J. E., Ross, S. A., 1985. A theory of the term structure of interest rates. Econometrica 53, 385-408. 
Derman, E., Kani, I., 1994. Riding on the smile. RISK 7, 32-39.

Duffie, D., Pan, J., Singleton, K. J., 2000. Transform analysis and asset pricing for affine jump-diffusions. Econometrica 68, 1343-1376.

Dupire, B., 1993. Model art. RISK September, 118-120.

Dupire, B., 1994. Pricing with a smile. RISK 7, 18-20.

Durrleman, V., 2004. From implied to spot volatilities. Ph.D. thesis, Princeton University.

Egloff, D., Leippold, M., Wu, L., 2010. The term structure of variance swap rates and optimal variance swap investments. Journal of Financial and Quantitative Analysis 45, 1279-1310.

Eraker, B., 2004. Do stock prices and volatility jump? Reconciling evidence from spot and option prices. The Journal of Finance 59, 1367-1404.

Gatheral, J., 2008. Developments in volatility derivatives pricing. Tech. rep., Baruch College, CUNY.

Harrison, M., Kreps, D., 1979. Martingales and arbitrage in multiperiod securities markets. Journal of Economic Theory 20, 381-408.

Harrison, M., Pliska, S., 1981. Martingales and stochastic integrals in the theory of continuous trading. Stochastic Processes and Their Applications 11, 215-260.

Heath, D., Jarrow, R., Morton, A., 1992. Bond pricing and the term structure of interest rates: A new methodology for contingent claims evaluation. Econometrica 60, 77-105.

Heston, S., 1993. A closed-form solution for options with stochastic volatility with applications to bonds and currency options. Review of Financial Studies 6, 327-343.

Hull, J., White, A., 1987. The pricing of options on assets with stochastic volatilities. The Journal of Finance 42, 281-300.

Jones, C. S., 2003. The dynamics of stochastic volatility: Evidence from underlying and options markets. Journal of Econometrics 116, 181-224.

Ledoit, O., Santa-Clara, P., Yan, S., 2002. Relative pricing of options with stochastic volatility. Tech. rep., University of California at Los Angeles.

Li, G., Zhang, C., 2010. On the number of state variables in options pricing. Management Science 55, 2058-2075.

Li, G., Zhang, C., 2013. Diagnosing affine models of options pricing: Evidence from VIX. Journal of Financial Economics 107, 199-219. 
Li, M., Pearson, N. D., Poteshman, A. M., 2004. Conditional estimation of diffusion processes. Journal of Financial Economics 74, 31-66.

Litterman, R., Scheinkman, J., 1991. Common factors affecting bond returns. Journal of Fixed Income June, 54-61.

Lyons, T. J., 1997. Derivatives as tradable assets. Risk 10th Anniversary Global Summit, 18-19.

Merton, R. C., 1973. The theory of rational option pricing. Bell Journal of Economics and Management Science 4, 141-183.

Nelson, D. B., 1990. ARCH models as diffusion approximations. Journal of Econometrics 45, 7-38.

Pan, J., 2002. The jump-risk premia implicit in options: Evidence from an integrated time-series study. Journal of Financial Economics 63, 3-50.

Rosenberg, J. V., 2000. Implied volatility functions: A reprise. Journal of Derivatives 8, 51-64.

Schönbucher, P. J., 1999. A market model of stochastic implied volatility. Philosophical Transactions of the Royal Society A 357, 2071-2092.

Stein, E. M., Stein, J. C., 1991. Stock price distributions with stochastic volatility: An analytic approach. Review of Financial Studies 4, 727-752.

Vuong, Q. H., 1989. Likelihood ratio tests for model selection and non-nested hypotheses. Econometrica 57 (2), 307-333. 
Panel A: Descriptive statistics for univariate time series

\begin{tabular}{lrrrr} 
& Mean & Std. Dev. & Skewness & Kurtosis \\
\hline \hline Variance swap rates & & & & \\
\hline 2 months & 0.048 & 0.0300 & 2.026 & 9.759 \\
3 months & 0.048 & 0.031 & 1.867 & 8.926 \\
6 months & 0.050 & 0.031 & 1.860 & 9.357 \\
1 year & 0.053 & 0.030 & 1.403 & 6.438 \\
2 years & 0.056 & 0.029 & 1.127 & 4.604 \\
& & & & \\
VIX & & & & \\
\hline & 0.047 & 0.031 & 1.633 & 6.505 \\
S\&P 500 & & & & \\
\hline & 0.000 & 0.011 & -0.104 & 6.263
\end{tabular}

Panel B: Principal components

\begin{tabular}{lrrrrrr} 
& $1^{\text {st }}$ & \multicolumn{1}{c}{$2^{\text {nd }}$} & \multicolumn{1}{c}{$3^{\text {rd }}$} & \multicolumn{1}{c}{$4^{\text {th }}$} & \multicolumn{1}{c}{$5^{\text {th }}$} & \multicolumn{1}{c}{$6^{\text {th }}$} \\
\hline \hline Eigenvalues & & & & & & \\
\hline Eigenvectors & $93.09 \%$ & $6.13 \%$ & $0.62 \%$ & $0.08 \%$ & $0.05 \%$ & $0.02 \%$ \\
\hline & & & & & & \\
& 0.394 & -0.574 & 0.614 & 0.370 & 0.044 & 0.023 \\
& 0.412 & -0.357 & -0.176 & -0.734 & 0.352 & -0.093 \\
& 0.419 & -0.185 & -0.418 & 0.056 & -0.782 & -0.044 \\
& 0.420 & 0.131 & -0.459 & 0.438 & 0.444 & 0.455 \\
& 0.408 & 0.427 & 0.047 & 0.179 & 0.149 & -0.771 \\
& 0.396 & 0.556 & 0.453 & -0.311 & -0.209 & 0.433 \\
\hline
\end{tabular}

Table 1. Variance Swap Rates: Descriptive Statistics and Principal Components.

Notes: Sampling period January 4, 1996 to March 30, 2007. The number of daily observations is 2,827, excluding weekends and holidays. Data on variance swaps quotes are obtained from Bank of America. Principal components are extracted from the $6 \times 6$ covariance matrix taking $V I X^{2}$ as a proxy for the one month variance swap rate. 
Panel A: 2 months time-to-maturity reference variance swap rate

\begin{tabular}{|c|c|c|c|c|c|c|}
\hline & \multicolumn{2}{|c|}{ Heston-type diffusion } & \multicolumn{2}{|c|}{ GARCH-type diffusion } & \multicolumn{2}{|c|}{ CEV-type diffusion } \\
\hline & Estimate & Std.error & Estimate & Std.error & Estimate & Std.error \\
\hline \multicolumn{7}{|c|}{ Market-based model parameters } \\
\hline$\overline{\bar{\alpha}}$ & 0.101 & 0.002 & 0.125 & 0.005 & 0.075 & 0.002 \\
\hline$\lambda_{1}$ & -0.232 & 0.009 & -0.181 & 0.009 & -0.362 & 0.014 \\
\hline$\nu_{0}$ & 0.003 & 4.876 & 0.002 & 2.107 & -0.016 & 5.764 \\
\hline$\nu_{1}$ & -0.013 & 3.016 & 0.000 & 0.759 & -0.003 & 3.045 \\
\hline$\gamma_{1}$ & -0.784 & 0.012 & -0.832 & 0.009 & -0.791 & 0.014 \\
\hline$\gamma_{11}$ & 0.445 & 0.007 & 1.991 & 0.027 & 1.738 & 0.055 \\
\hline$\beta$ & 1 & - & 2 & - & 1.917 & 0.014 \\
\hline \multicolumn{7}{|c|}{ Pricing errors parameters } \\
\hline$\overline{\sigma_{3 M}^{2}}$ & 0.004 & 0.000 & 0.005 & 0.000 & 0.005 & 0.000 \\
\hline$\sigma_{6 M}^{2}$ & 0.017 & 0.001 & 0.016 & 0.001 & 0.021 & 0.002 \\
\hline$\sigma_{1 Y}^{2}$ & 0.025 & 0.001 & 0.035 & 0.002 & 0.049 & 0.005 \\
\hline
\end{tabular}

Panel B: 6 months time-to-maturity reference variance swap rate

\begin{tabular}{|c|c|c|c|c|c|c|}
\hline & \multicolumn{2}{|c|}{ Heston-type diffusion } & \multicolumn{2}{|c|}{ GARCH-type diffusion } & \multicolumn{2}{|c|}{ CEV-type diffusion } \\
\hline & Estimate & Std.error & Estimate & Std.error & Estimate & Std.error \\
\hline \multicolumn{7}{|c|}{ Market-based model parameters } \\
\hline $\bar{\alpha}$ & 0.133 & 0.005 & 0.127 & 0.004 & 0.097 & 0.002 \\
\hline$\lambda_{1}$ & -0.142 & 0.008 & -0.149 & 0.007 & -0.223 & 0.007 \\
\hline$\nu_{0}$ & -0.002 & 7.652 & -0.010 & 2.047 & -0.002 & 4.943 \\
\hline$\nu_{1}$ & 0.001 & 1.982 & 0.001 & 0.715 & -0.005 & 2.972 \\
\hline$\gamma_{1}$ & -0.41 & 0.037 & -0.851 & 0.006 & -0.812 & 0.010 \\
\hline$\gamma_{11}$ & 0.223 & 0.003 & 1.138 & 0.015 & 0.459 & 0.014 \\
\hline$\beta$ & 1 & - & 2 & - & 1.457 & 0.019 \\
\hline \multicolumn{7}{|c|}{ Pricing errors parameters } \\
\hline$\overline{\sigma_{2 M}^{2}}$ & 0.018 & 0.001 & 0.015 & 0.001 & 0.012 & 0.000 \\
\hline$\sigma_{3 M}^{2}$ & 0.007 & 0.000 & 0.007 & 0.000 & 0.007 & 0.000 \\
\hline$\sigma_{1 Y}^{2}$ & 0.006 & 0.000 & 0.007 & 0.000 & 0.006 & 0.000 \\
\hline
\end{tabular}

Table 2. Parameter Estimates for the 1-VS Model.

Notes: Sampling period January 4, 1996 to March 30, 2007. The number of daily observations is 2,827, excluding weekends and holidays. Data on variance swaps quotes are obtained from Bank of America. Standard errors have been calculated by evaluating the sample means of the cross products of the score functions, which in turn have been computed by numeric differentiation of the log likelihood. The models and parametrizations are given in Section 3. 


\begin{tabular}{lrrrr}
\multirow{2}{*}{\begin{tabular}{l} 
reference VS rate \\
\cline { 2 - 5 }
\end{tabular}} & \multicolumn{4}{c}{ Affine $P$-drift $(\delta=1)$} \\
\cline { 2 - 5 } & \multicolumn{2}{c}{ Full sample } & \multicolumn{2}{c}{ Sub sample } \\
\cline { 2 - 5 } & Estimate & Std.error & Estimate & Std.error \\
\hline \hline Market-based model parameters & 0.101 & 0.002 & 0.073 & 0.001 \\
\hline $\bar{\alpha}$ & -0.232 & 0.009 & -1.817 & 0.044 \\
$\lambda_{1}$ & 0.003 & 4.876 & -0.001 & 6.957 \\
$\nu_{0}$ & -0.013 & 3.016 & -0.018 & 3.716 \\
$\nu_{1}$ & -0.784 & 0.012 & -0.820 & 0.013 \\
$\gamma_{1}$ & 0.445 & 0.007 & 0.567 & 0.011 \\
$\gamma_{11}$ & & & & \\
Pricing errors parameters & 0.004 & 0.000 & 0.005 & 0.000 \\
\hline$\sigma_{3 M}^{2}$ & 0.017 & 0.001 & 0.024 & 0.003 \\
$\sigma_{6 M}^{2}$ & 0.025 & 0.001 & 0.036 & 0.003 \\
$\sigma_{1 Y}^{2}$ & 26.893 & \multicolumn{3}{c}{13.038} \\
\hline Log-likelihood & \multicolumn{5}{c}{}
\end{tabular}

\begin{tabular}{|c|c|c|c|c|}
\hline \multirow{3}{*}{$\begin{array}{l}2 \text { months time-to-maturity } \\
\text { reference VS rate }\end{array}$} & \multicolumn{4}{|c|}{ Constant market price of risk $(\delta=0)$} \\
\hline & \multicolumn{2}{|c|}{ Full sample } & \multicolumn{2}{|c|}{ Sub sample } \\
\hline & Estimate & Std.error & Estimate & Std.error \\
\hline \multicolumn{5}{|c|}{ Market-based model parameters } \\
\hline $\bar{\alpha}$ & 0.107 & 0.003 & 0.073 & 0.001 \\
\hline$\lambda_{1}$ & -0.220 & 0.009 & -1.817 & 0.044 \\
\hline$\nu_{0}$ & 0.014 & 1.913 & -0.018 & 3.658 \\
\hline$\nu_{1}$ & 0.003 & 0.785 & -0.001 & 1.082 \\
\hline$\gamma_{1}$ & -0.793 & 0.011 & -0.820 & 0.013 \\
\hline$\gamma_{11}$ & 0.447 & 0.007 & 0.567 & 0.011 \\
\hline \multicolumn{5}{|l|}{ Pricing errors parameters } \\
\hline$\sigma_{3 M}^{2}$ & 0.005 & 0.000 & 0.005 & 0.000 \\
\hline$\sigma_{6 M}^{2}$ & 0.019 & 0.001 & 0.024 & 0.003 \\
\hline$\sigma_{1 Y}^{2}$ & 0.026 & 0.001 & 0.036 & 0.003 \\
\hline Log-likelihood & \multicolumn{2}{|c|}{26.924} & \multicolumn{2}{|c|}{13.038} \\
\hline
\end{tabular}

Table 3. Parameter Estimates for the 1-VS Model: Different Drifts.

Notes: Full sample corresponds to the period January 4, 1996 to March 30, 2007 while subsample starts in October 10, 1997 and ends in September 30, 2003. The number of daily observations are 2,827 and 1,500, excluding weekends and holidays, respectively. Data on variance swaps quotes are obtained from Bank of America. Standard errors have been calculated by evaluating the sample means of the cross products of the score functions, which in turn have been computed by numeric differentiation of the log likelihood. The models and parametrizations are given in Section 3. 


\begin{tabular}{|c|c|c|c|c|}
\hline \multirow{3}{*}{$\begin{array}{l}2 \text { months and } 6 \text { months } \\
\text { time-to-maturity } \\
\text { reference VS rates }\end{array}$} & \multicolumn{4}{|c|}{ Affine $P$-drift $(\delta=1)$} \\
\hline & \multicolumn{2}{|c|}{ Full sample } & \multicolumn{2}{|c|}{ Sub sample } \\
\hline & Estimate & Std.error & Estimate & Std.error \\
\hline \multicolumn{5}{|c|}{ Market-based model parameters } \\
\hline $\bar{\alpha}$ & 0.129 & 0.013 & 0.095 & 0.003 \\
\hline$\lambda_{1}$ & -0.119 & 0.015 & -0.617 & 0.056 \\
\hline$\lambda_{2}$ & -6.028 & 0.086 & -5.095 & 0.149 \\
\hline$\nu_{0}$ & -0.016 & 7.636 & -0.005 & 11.748 \\
\hline$\nu_{1}$ & 0.002 & 2.515 & 0.003 & 3.187 \\
\hline$\nu_{2}$ & -0.738 & 3.468 & -0.282 & 3.929 \\
\hline$\gamma_{1}$ & -0.213 & 0.059 & -0.113 & 0.093 \\
\hline$\gamma_{2}$ & 0.022 & 0.039 & -0.065 & 0.065 \\
\hline$\gamma_{11}$ & 0.197 & 0.002 & 0.234 & 0.004 \\
\hline$\gamma_{22}$ & 0.353 & 0.009 & 0.444 & 0.016 \\
\hline$\gamma_{12}$ & 0.254 & 0.006 & 0.297 & 0.009 \\
\hline \multicolumn{5}{|l|}{ Pricing errors parameters } \\
\hline$\sigma_{3 M}^{2}$ & 0.002 & 0.000 & 0.002 & 0.000 \\
\hline$\sigma_{1 Y}^{2}$ & 0.004 & 0.000 & 0.004 & 0.000 \\
\hline Log-likelihood & \multicolumn{2}{|c|}{43.634} & \multicolumn{2}{|c|}{21.429} \\
\hline
\end{tabular}

\begin{tabular}{|c|c|c|c|c|}
\hline \multirow{3}{*}{$\begin{array}{l}2 \text { months and } 6 \text { months } \\
\text { time-to-maturity } \\
\text { reference VS rates }\end{array}$} & \multicolumn{4}{|c|}{ Constant market prices of risk $(\delta=0)$} \\
\hline & \multicolumn{2}{|c|}{ Full sample } & \multicolumn{2}{|c|}{ Sub sample } \\
\hline & Estimate & Std.error & Estimate & Std.error \\
\hline \multicolumn{5}{|c|}{ Market-based model parameters } \\
\hline $\bar{\alpha}$ & 0.129 & 0.013 & 0.095 & 0.003 \\
\hline$\lambda_{1}$ & -0.114 & 0.015 & -0.616 & 0.056 \\
\hline$\lambda_{2}$ & -6.022 & 0.086 & -5.121 & 0.149 \\
\hline$\nu_{0}$ & -0.016 & 7.636 & -0.006 & 5.572 \\
\hline$\nu_{1}$ & 0.002 & 2.515 & 0.002 & 0.949 \\
\hline$\nu_{2}$ & -0.738 & 3.468 & -0.281 & 1.256 \\
\hline$\gamma_{1}$ & -0.213 & 0.059 & -0.113 & 0.094 \\
\hline$\gamma_{2}$ & 0.022 & 0.039 & -0.066 & 0.066 \\
\hline$\gamma_{11}$ & 0.197 & 0.002 & 0.236 & 0.004 \\
\hline$\gamma_{22}$ & 0.353 & 0.009 & 0.442 & 0.016 \\
\hline$\gamma_{12}$ & 0.254 & 0.006 & 0.298 & 0.009 \\
\hline \multicolumn{5}{|l|}{ Pricing errors parameters } \\
\hline$\sigma_{3 M}^{2}$ & 0.002 & 0.000 & 0.002 & 0.000 \\
\hline$\sigma_{1 Y}^{2}$ & 0.004 & 0.000 & 0.004 & 0.000 \\
\hline Log-likelihood & \multicolumn{2}{|c|}{43.377} & \multicolumn{2}{|c|}{21.428} \\
\hline
\end{tabular}

Table 4. Parameter Estimates for the 2-VS Model: Different Drifts.

Notes: Full sample corresponds to the period January 4, 1996 to March 30, 2007 while subsample starts in October 10, 1997 and ends September 30, 2003. The number of daily observations are 2,827 and 1,500, excluding weekends and holidays, respectively. Data on variance swaps quotes are obtained from Bank of America. Standard errors have been calculated by evaluating the sample means of the cross products of the score functions, which in turn have been computed by numeric differentiation of the log likelihood. The models and parametrizations are given in Section 3. 
Panel A: 1-VS, 2 months time-to-maturity reference variance swap rate Additional VS rates

\begin{tabular}{|c|c|c|c|c|c|c|}
\hline & \multicolumn{2}{|c|}{ Reference VS only } & \multicolumn{2}{|c|}{$3,6 \& 12$ months } & \multicolumn{2}{|c|}{ All data } \\
\hline & Estimate & Std.error & Estimate & Std.error & Estimate & Std.error \\
\hline \multicolumn{7}{|c|}{ Market-based model parameters } \\
\hline$\overline{\bar{\alpha}}$ & 0.096 & 0.115 & 0.101 & 0.002 & 0.103 & 0.002 \\
\hline$\lambda_{1}$ & -0.762 & 0.986 & -0.232 & 0.009 & -0.195 & 0.006 \\
\hline$\nu_{0}$ & -0.002 & 5.113 & 0.003 & 4.876 & -0.010 & 5.142 \\
\hline$\nu_{1}$ & 0.001 & 3.563 & -0.013 & 3.016 & -0.004 & 2.942 \\
\hline$\gamma_{1}$ & -0.762 & 0.018 & -0.784 & 0.012 & -0.762 & 0.014 \\
\hline$\gamma_{11}$ & 0.444 & 0.010 & 0.445 & 0.007 & 0.439 & 0.007 \\
\hline \multicolumn{7}{|c|}{ Pricing errors parameters } \\
\hline$\overline{\sigma_{1 M}^{2}}$ & & & & & 0.009 & 0.000 \\
\hline$\sigma_{3 M}^{2}$ & & & 0.004 & 0.000 & 0.004 & 0.000 \\
\hline$\sigma_{6 M}^{2}$ & & & 0.017 & 0.001 & 0.023 & 0.002 \\
\hline$\sigma_{1 Y}^{2}$ & & & 0.025 & 0.001 & 0.023 & 0.001 \\
\hline$\sigma_{2 Y}^{2}$ & & & & & 0.050 & 0.005 \\
\hline
\end{tabular}

Panel B: 2-VS, 2 and 6 months time-to-maturity reference variance swap rates Additional VS rates

\begin{tabular}{|c|c|c|c|c|c|c|}
\hline & \multicolumn{2}{|c|}{ Reference VS only } & \multicolumn{2}{|c|}{$3 \& 12$ months } & \multicolumn{2}{|c|}{ All data } \\
\hline & Estimate & Std.error & Estimate & Std.error & Estimate & Std.error \\
\hline \multicolumn{7}{|c|}{ Market-based model parameters } \\
\hline $\bar{\alpha}$ & 0.031 & 0.124 & 0.129 & 0.013 & 0.140 & 0.008 \\
\hline$\lambda_{1}$ & -0.394 & 2.050 & -0.11 & 0.015 & -0.068 & 0.006 \\
\hline$\lambda_{2}$ & -3.023 & 3.143 & -6.02 & 0.086 & -5.749 & 0.048 \\
\hline$\nu_{0}$ & -0.008 & 6.845 & -0.016 & 7.636 & -0.009 & 7.163 \\
\hline$\nu_{1}$ & 0.001 & 5.400 & 0.002 & 2.515 & 0.001 & 2.769 \\
\hline$\nu_{2}$ & -1.997 & 3.072 & -0.738 & 3.468 & -0.246 & 3.371 \\
\hline$\gamma_{1}$ & -0.526 & 0.037 & -0.213 & 0.059 & -0.357 & 0.048 \\
\hline$\gamma_{2}$ & -0.043 & 0.045 & 0.022 & 0.039 & -0.033 & 0.047 \\
\hline$\gamma_{11}$ & 0.211 & 0.008 & 0.197 & 0.002 & 0.196 & 0.002 \\
\hline$\gamma_{22}$ & 0.305 & 0.015 & 0.353 & 0.009 & 0.36 & 0.008 \\
\hline$\gamma_{12}$ & 0.246 & 0.011 & 0.254 & 0.006 & 0.268 & 0.006 \\
\hline \multicolumn{7}{|c|}{ Pricing errors parameters } \\
\hline$\overline{\sigma_{1 M}^{2}}$ & & & & & 0.008 & 0.000 \\
\hline$\sigma_{3 M}^{2}$ & & & 0.002 & 0.000 & 0.002 & 0.000 \\
\hline$\sigma_{1 Y}^{2}$ & & & 0.004 & 0.000 & 0.004 & 0.000 \\
\hline$\sigma_{2 Y}^{2}$ & & & & & 0.009 & 0.001 \\
\hline
\end{tabular}

Table 5. Parameter Estimates for the 1-VS and 2-VS Models: Additional Variance Swaps Rates.

Notes: Sampling period January 4, 1996 to March 30, 2007. The number of daily observations is 2,827, excluding weekends and holidays. Data on variance swaps quotes are obtained from Bank of America. Standard errors have been calculated by evaluating the sample means of the cross products of the score functions, which in turn have been computed by numeric differentiation of the log likelihood. The models and parametrizations are given in Section 3. 


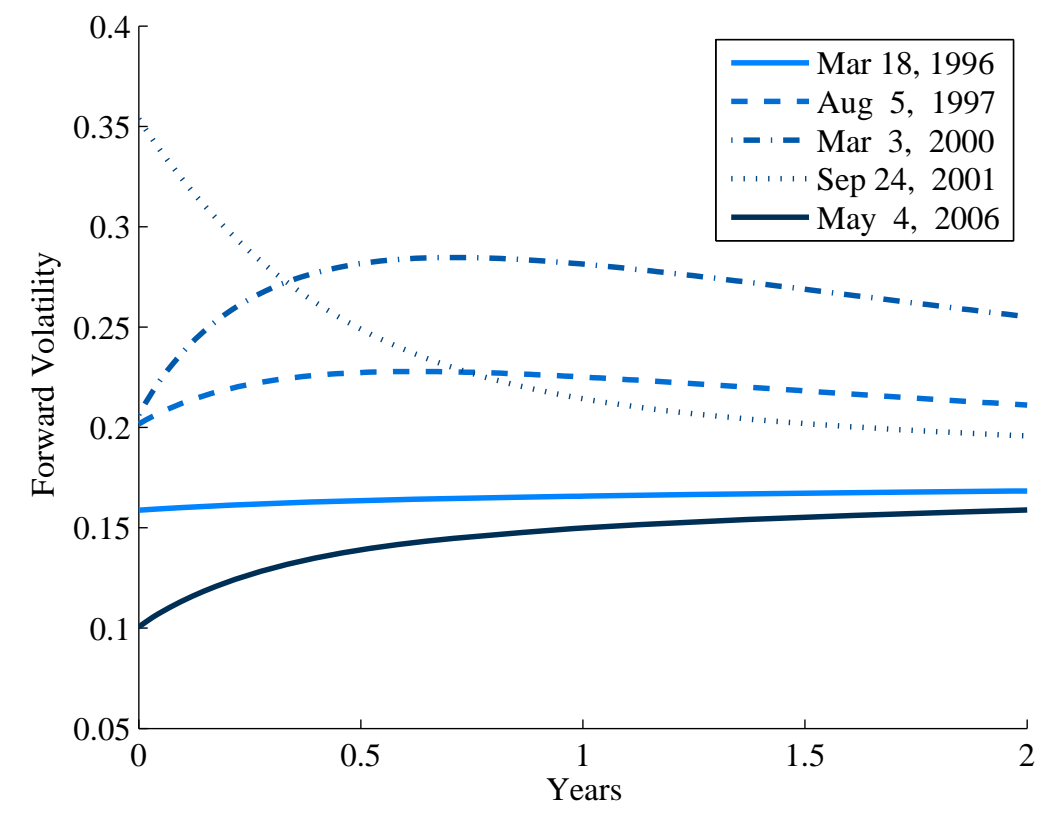

Fig. 1. Forward Volatility Curves.

Notes: These curves describe the conditional expectation at time $t$ of the instantaneous volatility at time $t+\tau$ (where $\tau$ is in the horizontal axis); they are defined by $\sqrt{G\left(y_{1}, y_{2}, \tau\right)}$ where

$$
G\left(y_{1}, y_{2}, \tau\right)=\left[1-\xi_{1}\left(\lambda_{1}, \lambda_{2} \tau\right)-\xi_{2}\left(\lambda_{1}, \lambda_{2} \tau\right)\right] \bar{\alpha}+\xi_{1}\left(\lambda_{1}, \lambda_{2} \tau\right) y_{1}+\xi_{2}\left(\lambda_{1}, \lambda_{2} \tau\right) y_{2}
$$

where the expressions for $\xi_{i}($.$) 's are given in Corollary 2$ in Section 2.4.2. The reference variance swap rates are 2 and 6 months time to maturity. The parameter values are $\lambda_{1}=-0.5, \lambda_{2}=-6$ and $\bar{\alpha}=0.04$. 


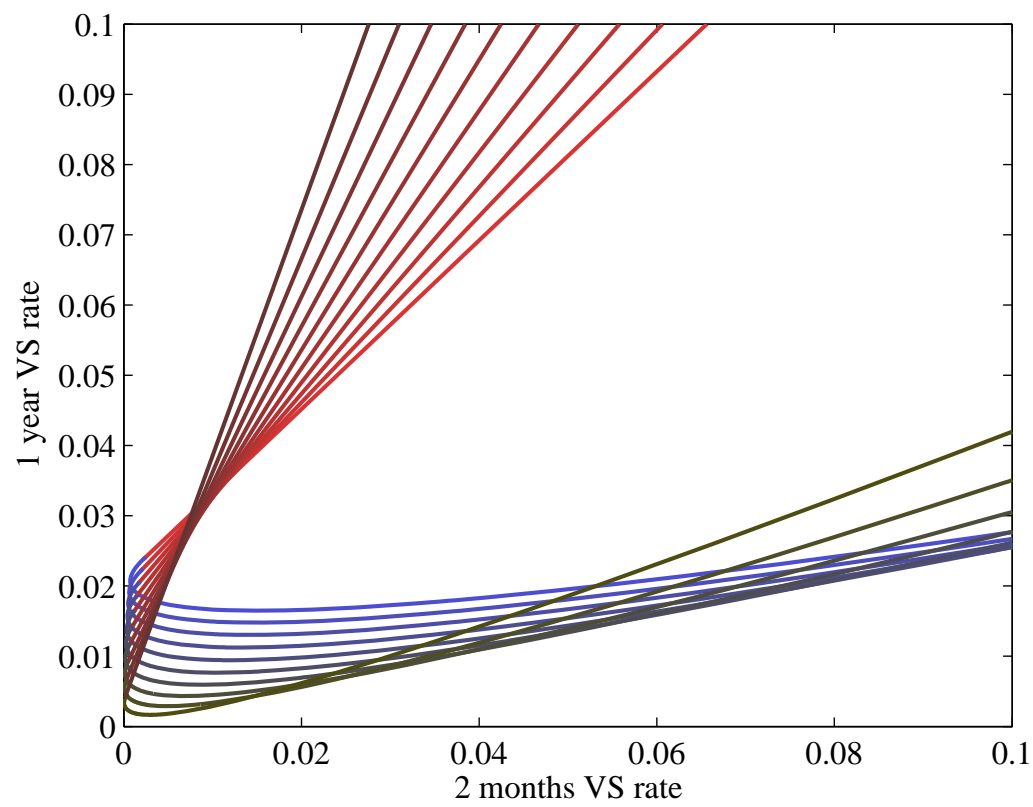

Fig. 2. Positivity Constraints.

Notes: To identify the region in the $y$-hyperplane for which $G\left(y_{1}, y_{2}, s\right) \geq 0$ consider a given value of $y=$ $\left(y_{1}, y_{2}\right)$ such that $G\left(y_{1}, y_{2}, s\right)=0$ defines a straight line in the $\mathbb{R}^{2}$-hyperplane. To determine the envelope i.e. set of $y$ 's, as a function of $s$, such that $G(y, s)$ remains zero for small variations of $s$, we should impose $\partial G(y, s) / \partial s=0$ by solving

$$
\left\{\begin{array}{c}
\left(1-\xi_{1}-\xi_{2}\right) \bar{\alpha}+\xi_{1} \cdot y_{1}+\xi_{2} \cdot y_{2}=0 \\
\left(1-\partial \xi_{1} / \partial s-\partial \xi_{2} / \partial s\right) \bar{\alpha}+\partial \xi_{1} / \partial s \cdot y_{1}+\partial \xi_{2} / \partial s \cdot y_{2}=0
\end{array}\right.
$$

The first equation defines a straight line in $\mathbb{R}^{2}$ such that in any neighborhood of the solution we will find positive and negative forward variances. In contrast, the second equation guarantees that we remain on the frontier as we move in $\mathbb{R}^{2}$. Different curves correspond to different parameter values of $\bar{\alpha}, \lambda_{1}$ and $\lambda_{2}$. Red lines correspond to $G\left(y_{1}, y_{2}, 0\right)=0$, while the grey dotted curves to $G\left(y_{1}, y_{2}, s\right)=0$ for $s>0$. 


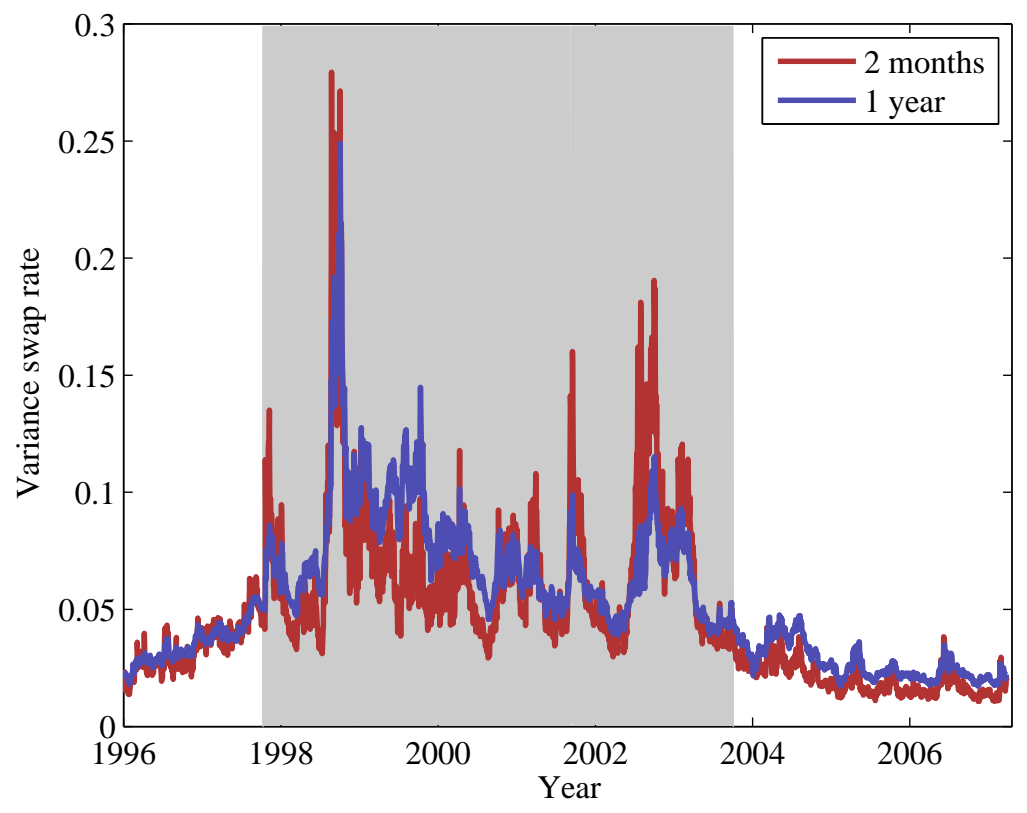

Fig. 3. Variance Swap Time Series: Full Sample and Subsample.

Notes: Full sample corresponds to the period January 4, 1996 to March 30, 2007 while subsample starts in October 10, 1997 and ends September 30, 2003. The shaded region represents the subsample. The number of daily observations are 2,827 and 1,500, excluding weekends and holidays, respectively. Data on variance swaps quotes are obtained from Bank of America. Gaussian kernel and Silverman's rule of thumb were used to compute the density. 
Panel A: In-Sample Pricing Errors

3-months maturity, 1-VS model

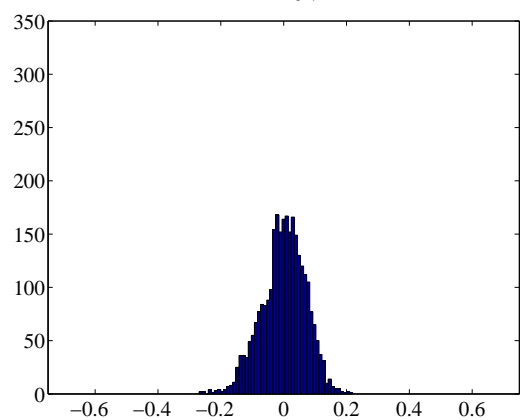

1-year maturity, 1-VS model

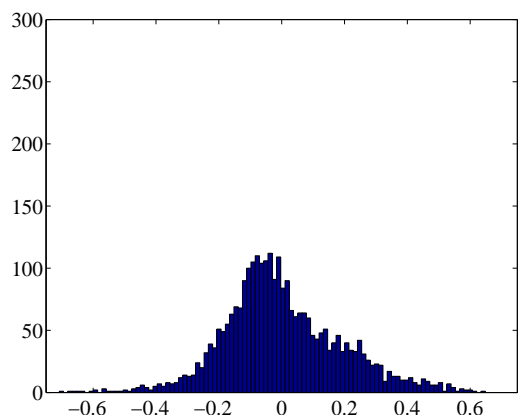

3-months maturity, 2-VS model

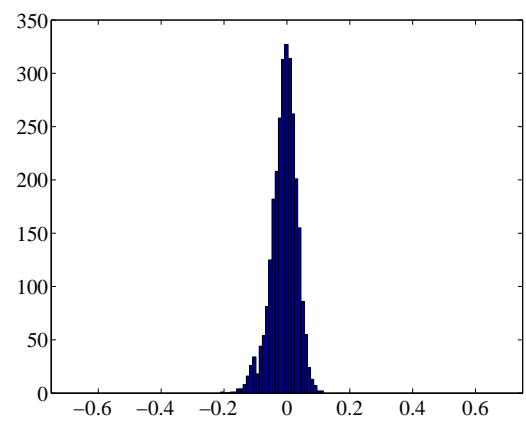

1-year maturity, 2-VS model

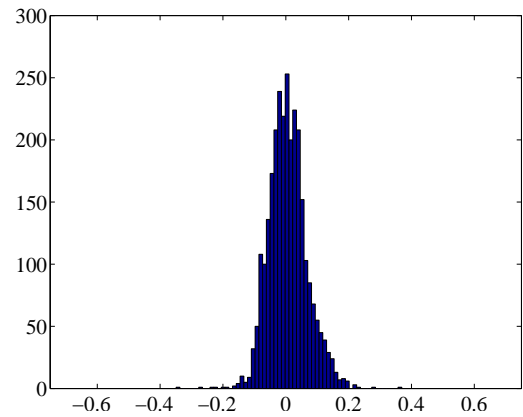

Panel B: Out-of-Sample Pricing Errors

1-month maturity, 1-VS model

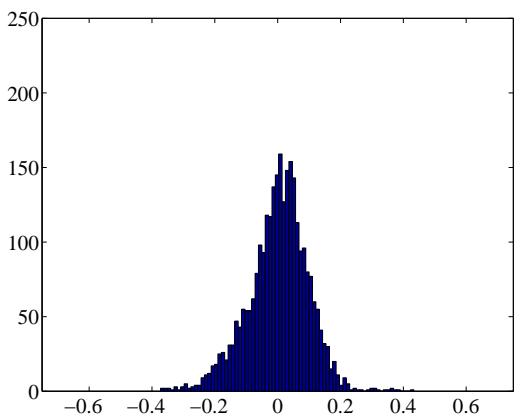

2-years maturity, 1-VS model

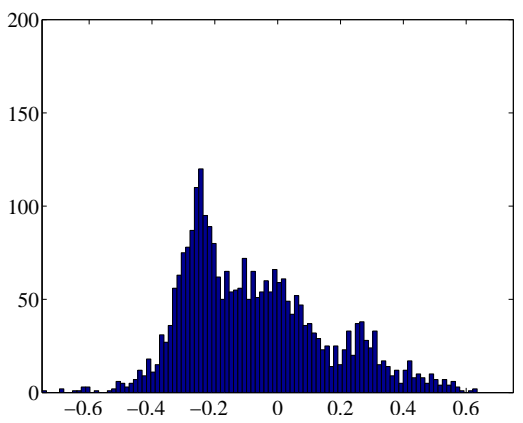

1-month maturity, 2-VS model

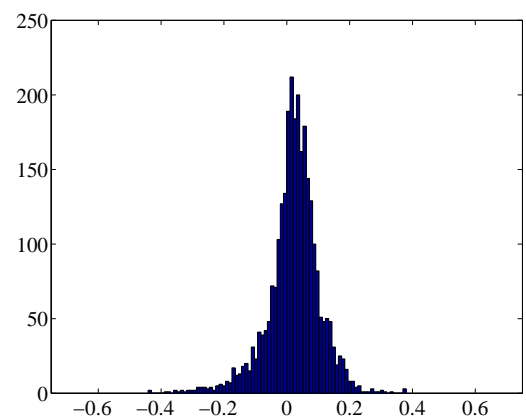

2-years maturity, 2-VS model

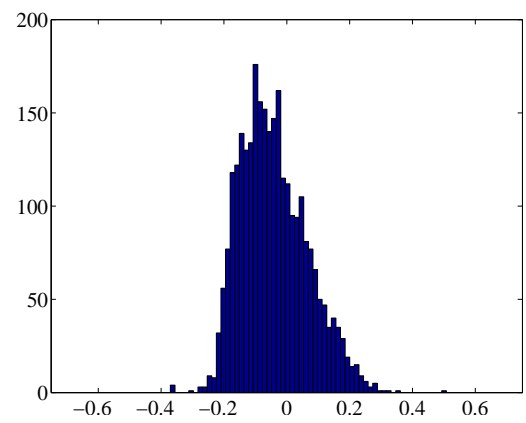

Figure 4: In-Sample and Out-of-Sample Pricing Errors

Notes: Sampling period January 4, 1996 to March 30, 2007. The number of daily observations is 2,827, excluding weekends and holidays. Data on variance swaps quotes are obtained from Bank of America. The models are given in Section 3. Both models are estimated using data on the spot and 2, 3, 6 and 12 months time to maturities. 1-VS (2-VS) model is estimated using 2 months (2 and 6 months) as reference rate. Both models are estimated under the maintained assumption that $\beta=\delta=1$. 


\section{Appendix 1: Proofs}

\section{A. Proof of Proposition 1}

Let $\mathcal{D}$ denote the infinitesimal operator for the multivariate diffusion $Y^{d}$ :

$$
\mathcal{D} G(y, \tau)=\sum_{i=1}^{d} \frac{\partial G(y, \tau)}{\partial y_{i}} \mu_{Y^{\tau_{i}}}^{Q}(y)+\frac{1}{2} \sum_{i, j=1}^{d} \frac{\partial^{2} G(y, \tau)}{\partial y_{i} \partial y_{j}}\left[\Sigma_{Y} \Sigma_{Y}^{\prime}\right]^{(i, j)}(y),
$$

where $y=\left(y_{1}, \ldots, y_{d}\right)^{\prime}$. To fully characterize the arbitrage-free model it is enough to solve the problem

$$
(P)\left\{\begin{array}{l}
\mathcal{D} G-\partial_{\tau} G=0 \text { in } \mathcal{Y} \times \mathbb{R}_{+} \\
\frac{1}{\tau_{j}} \int_{0}^{\tau_{j}} G\left(y_{j}, y_{-j}, \tau\right) d \tau=y_{j} \text { for all } y_{-j} \in \mathcal{Y}_{-j} \text { and } j=1, \ldots, d
\end{array}\right.
$$

that incorporates the boundary conditions from the definition of $Y^{d}$. Noting that by Itô's lemma,

$$
d u_{t}(\tau)=\mathcal{L} G(y, \tau) d t+\frac{\partial G(y, \tau)}{\partial y} \Sigma_{Y Y}(y) \cdot d W_{t}^{Q},
$$

and therefore, given that $\partial u_{t}(\tau) / \partial \tau=\partial G(y, \tau) / \partial \tau$, to avoid arbitrage (2.6) should hold.

\section{B. Proof of Proposition 2}

We start by stating the problem for $G$ under assumptions (A1) and (A2). After Proposition 1, consistent forward variance curves satisfy (2.6). However, under Assumption 1,

$$
\sum_{i, j=1}^{d} \frac{\partial^{2} G(y, \tau)}{\partial y_{i} \partial y_{j}}\left[\Sigma_{Y} \Sigma_{Y}^{\prime}\right]^{(i, j)}(y)=0 \quad \text { for all }(i, j)
$$

where $y=\left(y_{1}, \ldots, y_{d}\right)^{\prime}$. Moreover, under (A2) variance swap rates drifts are affine in variance swap rates. Hence $G$ can be characterized in terms of the following PDE:

$$
\frac{\partial G(y, \tau)}{\partial \tau}=\sum_{i=1}^{d} \frac{\partial G(y, \tau)}{\partial y_{i}}\left(\alpha_{0 i}+\sum_{j=1}^{d} \alpha_{j i} y\right)
$$

We use the Method of Characteristics to solve the PDE, which consists of characterizing curves (called characteristic curves) along which the PDE becomes an ordinary differential equation. Then, the solution of the ODE can be used to obtain a solution of the original PDE. Let $G$ be a function of the auxiliary variable $s$ : $G(s)=G\left(\tau(s), y_{1}(s), \ldots, y_{d}(s)\right)$. Taking derivatives of $G$ with respect to $s$ yields

$$
\frac{\partial G}{\partial s}=\frac{\partial G}{\partial \tau} \frac{\partial \tau}{\partial s}+\sum_{i=1}^{d} \frac{\partial G}{\partial y_{i}} \frac{\partial y_{i}}{\partial s}
$$

It is enough to find curves $\tau(s), y_{1}(s), \ldots, y_{d}(s)$ such that $G$ is constant (i.e. $\partial G / \partial s=0$ ) and along which the PDE is satisfied. Given the original PDE, the curves that characterize its solution are the ones that solve the following system of ODEs

$$
\left\{\begin{array}{cc}
\partial \tau / \partial s & =-1 \\
\partial y_{1} / \partial s & =\mu_{1}\left(y_{1}, \ldots, y_{d}\right) \\
\vdots & \vdots \\
\partial y_{d} / \partial s & =\mu_{d}\left(y_{1}, \ldots, y_{d}\right)
\end{array}\right.
$$


Since $\mu_{i}\left(y_{1}, \ldots, y_{d}\right)$ 's are affine in $y$, the system can be written in matrix form as

$$
\left[\begin{array}{l}
\dot{\tau} \\
\dot{y}
\end{array}\right]=\left[\begin{array}{ll}
0 & 0 \\
0 & A
\end{array}\right]\left[\begin{array}{l}
\tau \\
y
\end{array}\right]+\left[\begin{array}{c}
-1 \\
b
\end{array}\right]
$$

where

$$
A=\left[\begin{array}{ccc}
\alpha_{11} & \cdots & \alpha_{d 1} \\
\vdots & \ddots & \vdots \\
a_{1 d} & \cdots & \alpha_{d d}
\end{array}\right] \text { and } b=\left[\begin{array}{c}
\alpha_{01} \\
\vdots \\
\alpha_{0 d}
\end{array}\right]
$$

Given the separability of the system, we can solve for $\tau$ and $y$ separately. We have that

$$
\tau(s)=-\left(s-s_{0}\right)+K_{0 \tau}
$$

where $\tau\left(s_{0}\right)=K_{0 \tau} \in \mathbb{R}$. Without loss of generality we assume $s_{0}=0$ and $K_{0 \tau}=0$ so that $\tau=-s$. Regarding $y$, from basic ODEs theory, the general solution $y(s)=\left(y_{1}(s), \ldots, y_{d}(s)\right)^{\prime}$ can be written as

$$
y(s)=e^{A\left(s-s_{0}\right)} K_{0 y}+\int_{s_{0}}^{s} e^{A(s-r)} d r \cdot b
$$

where $K_{0 y} \in \mathbb{R}^{d}$ is such that $y\left(s_{0}\right)=y(0)=K_{0 y}$. Hence,

$$
K_{0 y}(s, y)=\left[e^{A s}\right]^{-1}\left[y-\int_{s_{0}}^{s} e^{A(s-r)} d r \cdot b\right]
$$

define $d$ curves implicitly which satisfy the system of ODEs and, by extension, the PDE. Notice that these $d$ characteristic functions are affine in $y$. It can be seen that any differentiable function that takes values on $K_{0 y_{1}}, \ldots, K_{0 y_{d}}$ into the real line satisfies the PDE. Indeed, letting

$$
F(\tau, y):=\tilde{F}\left(K_{0 y_{1}}(\tau, y), \ldots, K_{0 y_{d}}(\tau, y)\right)
$$

where $\tilde{F}: \mathbb{R} \times \mathbb{R}^{d} \rightarrow \mathbb{R}$. Then, by the chain rule we have

$$
\frac{\partial F}{\partial s}=\sum_{i=1}^{d} \frac{\partial \tilde{F}}{\partial K_{0 y_{i}}}\left[\frac{\partial K_{0 y_{i}}}{\partial \tau} \frac{\partial \tau}{\partial s}+\sum_{j=1}^{d} \frac{\partial K_{0 y_{i}}}{\partial y_{j}} \frac{\partial y_{j}}{\partial s}\right]
$$

and collecting terms on $\partial \tau / \partial s$ and $\partial y_{i} / \partial s$ :

$$
\frac{\partial F}{\partial s}=\left(\sum_{i=1}^{d} \frac{\partial \tilde{F}}{\partial K_{0 y_{i}}} \frac{\partial K_{0 y_{i}}}{\partial \tau}\right) \frac{\partial \tau}{\partial s}+\sum_{i=1}^{d}\left[\left(\sum_{j=1}^{d} \frac{\partial \tilde{F}}{\partial K_{0 y_{j}}} \frac{\partial K_{0 y_{j}}}{\partial y_{i}}\right) \frac{\partial y_{i}}{\partial s}\right] .
$$

Using the chain rule again, $\partial F / \partial .=\sum_{i=1}^{d} \partial \tilde{F} / \partial K_{0 y_{i}} \cdot \partial K_{0 y_{i}} / \partial$., and, since by construction $\partial F / \partial s=0$, $\partial \tau / \partial s=-1, \partial y_{i} / \partial s=\mu_{i}(y)$ for $i=1, \ldots, d$,

$$
0=-\frac{\partial F}{\partial \tau}+\sum_{i=1}^{d} \frac{\partial F}{\partial y_{i}} \mu_{i}(y)
$$

and the result follows. However, since the solution has to be affine in $y$, the only candidate is an affine function of the characteristic curves:

$$
G(\tau, y):=\sum_{i=1}^{d} M_{i} K_{0 i}(\tau, y)+M_{d+1} \text { where } M_{i} \in \mathbb{R}, i=1, \ldots, d+1
$$


It remains to impose that the integral boundary conditions are satisfied. Collecting terms in $y_{i}$ in the previous expression we can rewrite $G$ as

$$
G(\tau, y)=\sum_{i=1}^{d} \xi_{i}(\tau, \alpha, M) y_{i}+\xi_{d+1}\left(\tau, \alpha, \alpha_{0}, M\right),
$$

where $\alpha=\left(\alpha_{11}, \ldots, \alpha_{d d}\right)^{\prime}, \alpha_{0}=\left(\alpha_{01}, \ldots, \alpha_{0 d}\right)^{\prime}$ and $M=\left(M_{1}, \ldots, M_{d}\right)^{\prime}$. The boundary conditions can be written as:

$$
\begin{aligned}
\tau_{i}^{-1}\left[\Xi_{i}\left(\tau_{i}, \alpha, M\right)-\Xi_{i}(0, \alpha, M)\right] & =1, \text { for } i=1, \ldots, d \\
\tau_{i}^{-1}\left[\Xi_{j}\left(\tau_{i}, \alpha, M\right)-\Xi_{j}(0, \alpha, M)\right] & =0, \text { for } i \neq j, i, j=1, \ldots, d \\
\tau_{i}^{-1}\left[\Xi_{d+1}\left(\tau_{i}, \alpha, \alpha_{0}, M, M_{d+1}\right)-\Xi_{d+1}\left(0, \alpha, \alpha_{0}, M, M_{d+1}\right)\right] & =0, \text { for } i=1, \ldots, d
\end{aligned}
$$

where $\Xi_{i}(\tau, \alpha, M)=\int_{0}^{\tau} \xi_{i}(s, \alpha, M) d s$. Notice that there is a total of $(d+1) d$ restrictions and $(d+1)^{2}$ parameters. This leaves $d+1$ free parameters in the system. A natural choice of parameterization is $\lambda_{1}, \ldots, \lambda_{d}$ the eigenvalues of the system, which can be interpreted as speed of reversion parameters of the system, and $\bar{\alpha}$, which can be understood as the steady state level of the system. More precisely, notice that from the $d^{2}$ first set of restrictions, $\alpha$ and $M$ can be specified in term of $\lambda_{1}, \ldots, \lambda_{d}$. From the last set of $d$ restrictions, we pin down $\alpha_{0}$ and $M_{d+1}$ with $\lambda_{1}, \ldots, \lambda_{d}$ and one more parameter, $\bar{\alpha}$. Moreover, notice that

$$
\xi_{d+1}\left(\tau, \lambda_{1}, \ldots, \lambda_{d}, \bar{\alpha}\right)=\bar{\alpha}\left(1-\sum_{i=1}^{d} \xi_{i}\left(\tau, \lambda_{1}, \ldots, \lambda_{d}\right)\right)
$$

is the only compatible function with the whole set of restrictions.

Up to this point we have proved the existence of a solution for the problem. It only remains to prove uniqueness. To this end, we will assume the existence of another solution:

$$
H(\tau, y)=\sum_{i=1}^{d} \zeta_{i}(\tau) y_{i}+\zeta_{d+1}(\tau)
$$

Then, it must satisfy:

$$
\frac{\partial H(y, \tau)}{\partial \tau}=\sum_{i=1}^{d} \frac{\partial H(y, \tau)}{\partial y_{i}}\left(\alpha_{0 i}+\sum_{j=1}^{d} \alpha_{j i} y_{j}\right)
$$

where

$$
\frac{\partial H(y, \tau)}{\partial y_{i}}=\zeta_{i}(\tau) \text { and } \frac{\partial H(y, \tau)}{\partial \tau}=\sum_{i=1}^{d} \dot{\zeta}_{i}(\tau) y_{i}+\dot{\zeta}_{d+1}(\tau)
$$

Equating coefficients in $y_{i}$ we obtain that the following system of ODEs has to be true:

$$
\left\{\begin{aligned}
\dot{\zeta}_{i}(\tau) & =\sum_{j=1}^{d} \alpha_{j i} \zeta_{j}(\tau) \quad \text { for all } i \\
\dot{\zeta}_{d+1}(\tau) & =\sum_{i=1}^{d} \alpha_{0 i} \zeta_{i}(\tau)
\end{aligned}\right.
$$

or in matrix form, letting $\zeta=\left(\zeta_{1}, \ldots, \zeta_{d}\right)$,

$$
\left[\begin{array}{c}
\dot{\zeta} \\
\dot{\zeta}_{d+1}
\end{array}\right]=\left[\begin{array}{cc}
A & 0 \\
b^{\prime} & 0
\end{array}\right]\left[\begin{array}{c}
\zeta \\
\zeta_{d+1}
\end{array}\right]
$$

It is clear that this problem can be solved first for $\zeta$ and then for $\zeta_{d+1}$ and the general solution of this system is

$$
\zeta(\tau)=e^{A\left(\tau-\tau_{0}\right)} K_{0 \zeta}
$$


Let us assume, without loss of generality, that $\tau_{0}=0$. Then,

$$
\zeta_{d+1}(\tau)=b^{\prime} \int_{0}^{\tau} e^{A r} K_{0 \zeta} d r+C
$$

where $C$ is a constant. It is clear now that if we rename $K_{0 \zeta}=M, C=M_{d+1}$ the two solutions coincide: Taking into account the definition of $\xi_{i}$ and the fact that $\tau=-s$ we have

$$
\xi(\tau, \alpha, M)=\left[e^{A(-\tau)}\right]^{-1} M=e^{A \tau} M=e^{A \tau} K_{0 \zeta}=\zeta(\tau)
$$

and

$$
\begin{aligned}
\xi_{d+1}(\tau, \alpha, M)-M_{d+1} & =-M^{\prime}\left[e^{A(-\tau)}\right]^{-1}\left[\int_{0}^{-\tau} e^{A(-\tau-r)} d r\right] b \\
& =M^{\prime} e^{A \tau}\left[\int_{-\tau}^{0} e^{A(-\tau-r)} d r\right] b \\
& =b^{\prime}\left[\int_{0}^{\tau} e^{A r} d r\right] K_{0 \zeta} \\
& =\zeta_{d+1}(\tau)-C .
\end{aligned}
$$

\section{Proof of Corollary 1}

To show the result we start with the following conjecture: $G(y, \tau)=\left(y-k_{1}\right) k_{3} e^{k_{2}\left(\tau-\tau_{1}\right)}+k_{4}$, so that $k_{2}\left(y-k_{1}\right) k_{3} e^{k_{2}\left(\tau-\tau_{1}\right)}=\lambda_{1}(\alpha-y) k_{3} e^{k_{2}\left(\tau-\tau_{1}\right)}$ from where $k_{1}=\alpha$ and $k_{2}=-\lambda_{1}$. Then we can use $\frac{1}{\tau_{1}} \int_{0}^{\tau_{1}} G(y, \tau) d \tau=y$ to find $k_{3}$ and $k_{4}$. Specifically,

$$
\frac{1}{\tau_{1}} \int_{0}^{\tau_{1}} G(y, \tau) d \tau=k_{4}+(y-\alpha) k_{3} \frac{e^{\lambda_{1} \tau_{1}}-1}{\lambda_{1} \tau_{1}}=y
$$

for all $y \in \mathcal{Y}$ implies $k_{3}=\frac{\lambda_{1} \tau_{1}}{e^{\lambda_{1} \tau}-1}$ and $k_{4}=\alpha$. Hence

$$
G(y, \tau)=\frac{\lambda_{1} \tau_{1}}{e^{\lambda_{1} \tau_{1}}-1}(y-\alpha) e^{-\lambda_{1}\left(\tau-\tau_{1}\right)}+\alpha=\frac{\lambda_{1} \tau_{1}}{1-e^{-\lambda_{1} \tau_{1}}}(y-\alpha) e^{-\lambda_{1} \tau}+\alpha
$$

\section{Proof of Corollary 2}

It follows directly from Proposition 2 and the following two steps: First we have to find the general solution of the dynamic system implied by Proposition 2 and the second step involves finding the particular solution by imposing the integral boundary conditions.

Step 1: Taking derivatives of $G$ with respect to $s$ we get

$$
\frac{\partial G}{\partial s}=\frac{\partial G}{\partial \tau} \frac{\partial \tau}{\partial s}+\frac{\partial G}{\partial y_{1}} \frac{\partial y_{1}}{\partial s}+\frac{\partial G}{\partial y_{2}} \frac{\partial y_{2}}{\partial s} .
$$

The curves $\tau(s), y_{1}(s)$ and $y_{2}(s)$ along which $G$ is constant are characterized by the following system of ODEs

$$
\dot{\Phi}=A \Phi+b \text { with } A=\left[\begin{array}{ccc}
0 & 0 & 0 \\
0 & \alpha_{11} & \alpha_{21} \\
0 & \alpha_{12} & \alpha_{22}
\end{array}\right] \text { and } b=\left[\begin{array}{c}
-1 \\
\alpha_{01} \\
\alpha_{02}
\end{array}\right]
$$


where $\Phi=\left(\tau, y_{1}, y_{2}\right)^{\prime}$. The solution, $\Phi^{*}(s)=\left(\tau(s), y_{1}(s), y_{2}(s)\right)^{\prime}$, has the form

$$
\Phi^{*}(s)=e^{A\left(s-s_{0}\right)} K_{0}+\int_{s_{0}}^{s} e^{A(s-r)} d r \cdot b
$$

where $K_{0}$ and $s_{0}$ are such that $\Phi^{*}\left(s_{0}\right)=K_{0}$. Hence,

$$
K_{0}=\left[e^{A\left(s-s_{0}\right)}\right]^{-1}\left[\Phi(s)-\int_{s_{0}}^{s} e^{A(s-r)} d r \cdot b\right]
$$

defines three curves implicitly which satisfy the system of ODEs and, by extension, the PDE. The separability of the system of ODEs allows us, from now on, to focus on the subsystem of ODEs involving $y_{1}$ and $y_{2}$ taking into account the change of variable from $s$ to $\tau$. Without loss of generality we assume $K_{0 \tau}=0$ and $s_{0}=0$, so that the implicit curves defined by (D.1) are

$$
\begin{aligned}
& K_{0 y_{1}}\left(\tau, y_{1}, y_{2}\right)=a(\tau)\left[y_{1}-p_{1}(\tau)\right]+b(\tau)\left[y_{2}-p_{2}(\tau)\right] \\
& K_{0 y_{2}}\left(\tau, y_{1}, y_{2}\right)=c(\tau)\left[y_{1}-p_{1}(\tau)\right]+d(\tau)\left[y_{2}-p_{2}(\tau)\right]
\end{aligned}
$$

where

$$
\begin{aligned}
& a(\tau)=\frac{\left(\alpha_{11}-\lambda_{1}\right) e^{-\lambda_{2} \tau}-\left(\alpha_{11}-\lambda_{2}\right) e^{-\lambda_{1} \tau}}{\lambda_{2}-\lambda_{1}} \\
& b(\tau)=\frac{\alpha_{21}\left(e^{-\lambda_{2} \tau}-e^{-\lambda_{1} \tau}\right)}{\lambda_{2}-\lambda_{1}} \\
& c(\tau)=\frac{\alpha_{12}\left(e^{-\lambda_{2} \tau}-e^{-\lambda_{1} \tau}\right)}{\lambda_{2}-\lambda_{1}} \\
& d(\tau)=\frac{\left(\alpha_{11}-\lambda_{1}\right) e^{-\lambda_{1} \tau}-\left(\alpha_{11}-\lambda_{2}\right) e^{-\lambda_{2} \tau}}{\lambda_{2}-\lambda_{1}}
\end{aligned}
$$

and

$$
\begin{aligned}
p_{1}(\tau)= & \frac{\left.\alpha_{02} \alpha_{21}\left[\lambda_{2}\left(1-e^{\tau \lambda 1}\right)-\lambda_{1}\left(1-e^{\tau \lambda 2}\right)\right)\right]}{\left(\lambda_{2}-\lambda_{1}\right) \lambda_{1} \lambda_{2}} \\
p_{2}(\tau)= & \frac{\alpha_{01} \alpha_{12}\left[\lambda_{2}\left(1-e^{\tau \lambda 1}\right)-\lambda_{1}\left(1-e^{\tau \lambda 2}\right)\right]}{\left(\lambda_{2}-\lambda_{1}\right) \lambda_{1} \lambda_{2}} \\
& \left.\left.+\frac{\alpha_{02}\left[\left(1-e^{\tau \lambda 2}\right) \lambda_{1}\left(\alpha_{11}-\lambda_{2}\right)-\left(1-e^{\tau \lambda 1}\right) \lambda_{2}\left(\alpha_{11}-\lambda_{2}\right)-\left(1-e^{\tau \lambda 2}\right) \lambda_{1}\left(\alpha_{11}-\lambda_{1}\right)\right]}{\left(\lambda_{2}-\lambda_{1}\right) \lambda_{1} \lambda_{2}}\right)\right]
\end{aligned}
$$

where $\lambda_{1}=\left(\alpha_{22}+\alpha_{11}-\sqrt{D}\right) / 2$, and $\lambda_{2}=\left(\alpha_{22}+\alpha_{11}+\sqrt{D}\right) / 2$ with $D=\left(\alpha_{22}-\alpha_{11}\right)^{2}+4 \alpha_{12} \alpha_{21}$ are the eigenvalues that determine the dynamic properties of the system. Notice that $p_{1}(\tau)$ and $p_{2}(\tau)$ correspond to the particular solution of (D.1). In particular, if we rewrite the curves as follows:

$$
\begin{aligned}
& K_{0 y_{1}}\left(\tau, y_{1}, y_{2}\right)=a(\tau) y_{1}+b(\tau) y_{2}+e(\tau)+C_{1} \\
& K_{0 y_{2}}\left(\tau, y_{1}, y_{2}\right)=c(\tau) y_{1}+d(\tau) y_{2}+f(\tau)+C_{2}
\end{aligned}
$$

where

$$
\begin{aligned}
& e(\tau)=\left[\frac{\alpha_{02} \alpha_{21}+\alpha_{01}\left(\lambda_{1}-\alpha_{22}\right)}{\lambda_{1}-\lambda_{2}}\right] \frac{e^{-\lambda_{1} \tau}}{\lambda_{1}}-\left[\frac{\alpha_{02} \alpha_{21}+\alpha_{01}\left(\lambda_{2}-\alpha_{22}\right)}{\lambda_{1}-\lambda_{2}}\right] \frac{e^{-\lambda_{2} \tau}}{\lambda_{2}} \\
& f(\tau)=\left[\frac{\alpha_{02}\left(\lambda_{2}-\alpha_{22}\right)-\alpha_{01} \alpha_{12}}{\lambda_{2}-\lambda_{1}}\right] \frac{e^{-\lambda_{1} \tau}}{\lambda_{1}}+\left[\frac{\alpha_{01} \alpha_{12}+\alpha_{02}\left(\lambda_{1}-\alpha_{22}\right)}{\lambda_{2}-\lambda_{1}}\right] \frac{e^{-\lambda_{2} \tau}}{\lambda_{2}}
\end{aligned}
$$




$$
C_{1}=\frac{\alpha_{02} \alpha_{21}+\alpha_{01} \alpha_{22}}{\alpha_{11} \alpha_{22}-\alpha_{12} \alpha_{21}}, \quad \text { and } \quad C_{2}=\frac{\alpha_{02} \alpha_{11}-\alpha_{01} \alpha_{12}}{\alpha_{11} \alpha_{22}-\alpha_{12} \alpha_{21}}
$$

we get $\lim _{\tau \rightarrow \infty} K_{0 y_{1}}\left(\tau, y_{1}, y_{2}\right)=C_{1}$ and $\lim _{\tau \rightarrow \infty} K_{0 y_{2}}\left(\tau, y_{1}, y_{2}\right)=C_{2}$ provided the system is stationary, i.e. $\lambda_{1}>0$ and $\lambda_{2}>0$.

Step 2: Consider an affine solution, say

$$
\tilde{G}\left(K_{0 y_{1}}, K_{0 y_{2}}\right)=M_{1} \cdot K_{0 y_{1}}+M_{2} \cdot K_{0 y_{2}}+M_{3},
$$

so that

$$
\begin{aligned}
G\left(\tau, y_{1}, y_{2}\right)=M_{1}\left\{a(\tau)\left[y_{1}-p_{1}(\tau)\right]+b(\tau)\left[y_{2}-p_{2}(\tau)\right]\right\}+ \\
\\
+M_{2}\left\{c(\tau)\left[y_{1}-p_{1}(\tau)\right]+d(\tau)\left[y_{2}-p_{2}(\tau)\right]\right\}+M_{3} .
\end{aligned}
$$

Under this particular specification the final solution involves solving the following linear systems

$$
\begin{aligned}
& (i):\left\{\begin{array}{c}
\tau_{1}^{-1}\left[M_{1} A\left(\tau_{1}\right)+M_{2} C\left(\tau_{1}\right)\right]=1 \\
\tau_{2}^{-1}\left[M_{1} B\left(\tau_{2}\right)+M_{2} D\left(\tau_{2}\right)\right]=1
\end{array}\right. \\
& (i i):\left\{\begin{array}{c}
\tau_{2}^{-1}\left[M_{1} A\left(\tau_{2}\right)+M_{2} C\left(\tau_{2}\right)\right]=0 \\
\tau_{1}^{-1}\left[M_{1} B\left(\tau_{1}\right)+M_{2} D\left(\tau_{1}\right)\right]=0
\end{array}\right.
\end{aligned}
$$

and

$$
(i i i):\left\{\begin{array}{l}
\tau_{1}^{-1}\left\{M_{1}\left[E\left(\tau_{1}\right)+C_{1}\right]+M_{2}\left[F\left(\tau_{1}\right)+C_{2}\right]\right\}=-\tau_{1}^{-1} M_{3} \\
\tau_{2}^{-1}\left\{M_{1}\left[E\left(\tau_{2}\right)+C_{1}\right]+M_{2}\left[F\left(\tau_{2}\right)+C_{2}\right]\right\}=-\tau_{2}^{-1} M_{3}
\end{array}\right.
$$

where $X(\tau)=\int_{0}^{\tau} x(s) d s$. To do so, we first solve $(i)$ in order to obtain

$$
M_{1}=\frac{\tau_{2}^{-1} D\left(\tau_{2}\right)-\tau_{1}^{-1} C\left(\tau_{1}\right)}{\Delta} \quad \text { and } \quad M_{2}=\frac{\tau_{1}^{-1} A\left(\tau_{1}\right)-\tau_{2}^{-1} B\left(\tau_{2}\right)}{\Delta}
$$

where $\Delta=\tau_{1}^{-1} \tau_{2}^{-1}\left[A\left(\tau_{2}\right) D\left(\tau_{1}\right)-C\left(\tau_{2}\right) B\left(\tau_{1}\right)\right]$; and then, By imposing that the solution $\left(M_{1}, M_{2}\right)$ to system $(i)$ is also a solution of $(i i)$, together with $\lambda_{1} \lambda_{2}=\alpha_{11} \alpha_{22}-\alpha_{12} \alpha_{21}$ and $\lambda_{1}+\lambda_{2}=\alpha_{11}+\alpha_{22}$, we have a system of four equations in four unknowns that is satisfied by the following restrictions on the coefficients $\alpha_{11}, \alpha_{12}, \alpha_{21}$ and $\alpha_{22}$. Although there is another set of solutions for the $\alpha$ 's, this is the only one consistent with systems $(i)$ and $(i i)$ simultaneously. Imposing the above restrictions on $\left(M_{1}, M_{2}\right)$ we get

$$
\begin{aligned}
& M_{1}=\frac{\tau_{1} e^{\tau_{1}\left(\lambda_{1}+\lambda_{2}\right)}\left[e^{\tau_{2} \lambda_{1}} \lambda_{1}-e^{\tau_{2} \lambda_{2}} \lambda_{2}-e^{\tau_{2}\left(\lambda_{1}+\lambda_{2}\right)}\left(\lambda_{1}-\lambda_{2}\right)\right]}{\Upsilon\left(\lambda_{1}, \lambda_{2}, \tau_{1}, \tau_{2}\right)}, \quad \text { and } \\
& M_{2}=\frac{\tau_{2} e^{\tau_{2}\left(\lambda_{1}+\lambda_{2}\right)}\left[e^{\tau_{1} \lambda_{2}} \lambda_{2}-e^{\tau_{1} \lambda_{1}} \lambda_{1}-e^{\tau_{1}\left(\lambda_{1}+\lambda_{2}\right)}\left(\lambda_{2}-\lambda_{1}\right)\right]}{\Upsilon\left(\lambda_{1}, \lambda_{2}, \tau_{1}, \tau_{2}\right)}
\end{aligned}
$$

where

$$
\begin{aligned}
\Upsilon\left(\lambda_{1}, \lambda_{2}, \tau_{1}, \tau_{2}\right)= & e^{\tau_{1} \lambda_{1}+\tau_{2} \lambda_{2}}-e^{\tau_{2} \lambda_{1}+\tau_{1} \lambda_{2}}+e^{\tau_{2} \lambda_{1}+\tau_{1}\left(\lambda_{1}+\lambda_{2}\right)} \\
& -e^{\tau_{2} \lambda_{2}+\tau_{1}\left(\lambda_{1}+\lambda_{2}\right)}+e^{\tau_{1} \lambda_{2}+\tau_{2}\left(\lambda_{1}+\lambda_{2}\right)}-e^{\tau_{1} \lambda_{1}+\tau_{2}\left(\lambda_{1}+\lambda_{2}\right)}
\end{aligned}
$$

Finally, once we have determined $\left(M_{1}, M_{2}\right)$ and the $\alpha$ 's in terms of $\lambda_{1}, \lambda_{2}, \tau_{1}$ and $\tau_{2}$, system (iii) can be regarded as a linear system in $\left(\alpha_{01}, \alpha_{02}\right)$ from where we obtain the remaining drift parameters. 